\title{
Lattice Results for Low Moments of Light Meson Distribution Amplitudes
}

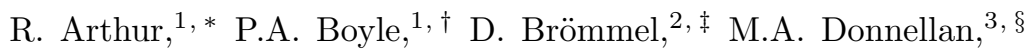 \\ J.M. Flynn, 2 , A. Jüttner,, ,** T.D. Rae, ${ }^{2, \dagger \dagger}$ and C.T.C. Sachrajda ${ }^{2, \dagger}$ \\ (RBC and UKQCD Collaborations) \\ ${ }^{1}$ SUPA, School of Physics, The University of Edinburgh, Edinburgh EH9 3JZ, UK \\ ${ }^{2}$ School of Physics and Astronomy, University of Southampton, Southampton SO17 1BJ, UK \\ ${ }^{3}$ NIC/DESY Zeuthen, Platanenallee 6, 15738 Zeuthen, Germany \\ ${ }^{4}$ CERN, Physics Department, 1211 Geneva 23, Switzerland
}

(Dated: January 10, 2011)

\begin{abstract}
As part of the UKQCD and RBC collaborations' $N_{f}=2+1$ domain-wall fermion phenomenology programme, we calculate the first two moments of the light-cone distribution amplitudes of the pseudoscalar mesons $\pi$ and $K$ and the (longitudinally-polarised) vector mesons $\rho, K^{*}$ and $\phi$. We obtain the desired quantities with good precision and are able to discern the expected quark-mass dependence of SU(3)-flavour breaking effects. An important ingredient of the calculation is the nonperturbative renormalisation of lattice operators using the $\mathrm{RI}^{\prime} / \mathrm{MOM}$ technique.
\end{abstract}

\section{INTRODUCTION}

Light-cone distribution amplitudes (DAs) are important nonperturbative quantities which (within the framework of collinear factorisation) parameterise in partonic terms the components of the hadronic wavefunction that control hard exclusive processes. Such processes provide hadron structure information complementary to that obtained from hard inclusive reactions.

The structure functions for inclusive processes are more accessible both experimentally and theoretically owing to their larger cross sections and branching ratios, simpler final-state detection and more straightforward factorisation properties. They do not specify the phases and correlations which would constitute amplitude-level hadron structure information, but probe instead the bound states' partonic content. Deep-inelastic scattering processes, for example, are controlled by the charge and momentum of the struck parton and are insensitive to its relation to the other hadronic constituents. The associated parton distribution functions (PDFs) are therefore single-particle probabilities, which reveal nothing about the role of particular Fock states or of correlations between quarks and gluons.

Distribution amplitudes, involved in exclusive processes, always appear in convolutions and, unlike the PDFs, are not directly measurable. These exclusive processes are dominated by specific partonic configurations. The outgoing quarks and gluons are unlikely to form a

\footnotetext{
* r.arthur@sms.ed.ac.uk

$\dagger$ paboyle@ph.ed.ac.uk

$\ddagger$ d.broemmel@fz-juelich.de Current address Jülich Supercomputing Centre, Institute for Advanced Simulation, Forschungszentrum Jülich GmbH, 52425 Jülich, Germany

$\S$ michael.donnellan@desy.de

ฯ j.m.flynn@soton.ac.uk

** juettner@mail.cern.ch

†† t.d.rae@phys.soton.ac.uk

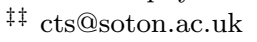

given final-state hadron unless either they are approximately collinear with small transverse separation, or one of the partons carries almost all of the hadron's momentum (the soft overlap or Feynman mechanism). In the former case, the basis for collinear factorisation [1], hard gluon exchange must occur to allow the struck or decaying parton to communicate with the others, turning them to the final direction. Since more partons require more hard gluons, exclusive cross-sections and decay rates are dominated by the valence Fock state at leading-order in $Q^{2}$, up to soft effects.

Hard exclusive processes are therefore controlled at leading-order by the distribution amplitudes of leadingtwist (an operator's twist is the difference between its dimension and its spin): essentially the overlap of the hadronic state with the valence Fock state in which, for a meson, the collinear quark-antiquark pair have small transverse separation and carry longitudinal momentum fractions $u$ and $\bar{u}=1-u$. The pion's electromagnetic form factor at large $Q^{2}$, for example, can be written as a convolution of distribution amplitudes $\phi_{\pi}\left(u, Q^{2}\right)$ for the incoming and outgoing pions with a perturbativelycalculable hard-scattering kernel. Higher-twist DAs associated with power-suppressed contributions originate in, for example, higher Fock states [2]. We consider only leading, twist-2, DAs in this paper.

The phenomenological importance of hard exclusive processes has grown since collinear factorisation was first established for cases such as the pion's electromagnetic form factor and the $\gamma \gamma^{*} \pi$ transition form factor around 30 years ago [1, 3 -5]. Of particular note is the theoretical description of hadronic $B$ decays, which have been studied in detail by BaBar and Belle and will be studied by $\mathrm{LHCb}$ and at super- $B$ factories in order to constrain the CKM matrix and to understand CP violation. Factorisation is more difficult to establish in $B$-physics because the hard collinear and soft mechanisms contribute at the same order in $1 / m_{b}$. Two approaches have been developed. In the QCD factorisation framework it has been shown that collinear factorisation can be applied to 
leading order in $1 / m_{b}$ to a large class of nonleptonic $B$ decays $[6-8]$. Soft-collinear effective theory (SCET) $[9$ 11] aims to provide a unified theoretical framework for the factorisation of both hard-collinear and soft effects. In both cases, distribution amplitudes play an important role as nonperturbative inputs in flavour physics.

In this paper we focus on the distribution amplitudes of the light pseudoscalar and longitudinally-polarised vector mesons, since, as we shall discuss in Sec. IB their lowest moments are of phenomenological interest and are calculable on the lattice. For pseudoscalars, these quantities are relevant for decays such as $B \rightarrow \pi \pi$ and $B \rightarrow \pi K$; they also appear in light-cone sum rule (LCSR) expressions for the form factors of semileptonic decays such as $B \rightarrow \pi l \nu$. For hard exclusive processes involving the light vector mesons $\rho, K^{*}$ and $\phi$, polarisation-dependence can reveal much about the underlying dynamics, with the longitudinally- and transversely-polarised final vector meson states often involving different aspects of weak interaction physics 12. Examples are the exclusive semileptonic $B \rightarrow \rho l \nu_{l}$, rare radiative $B \rightarrow \rho \gamma$ or nonleptonic, e.g. $B \rightarrow \pi \rho$, decays of $B$-mesons, which are important for extracting CKM matrix elements.

\section{A. Definitions}

Mesonic light-cone DAs are defined from meson-tovacuum matrix elements of quark-antiquark light-cone operators, which are non-local generalisations of those used to define the decay constants. For example, for pions

$$
\begin{gathered}
\left\langle 0\left|\bar{q}_{2}(z) \gamma_{\nu} \gamma_{5} \mathcal{P}(z,-z) q_{1}(-z)\right| \pi(p)\right\rangle_{z^{2}=0} \equiv \\
\quad i f_{\pi} p_{\nu} \int_{0}^{1} d u e^{i(u-\bar{u}) p \cdot z} \phi_{\pi}(u, \mu)
\end{gathered}
$$

and for longitudinally-polarised rho-mesons

$$
\begin{aligned}
& \left\langle 0\left|\bar{q}_{2}(z) \gamma_{\nu} \mathcal{P}(z,-z) q_{1}(-z)\right| \rho(p ; \lambda)\right\rangle_{z^{2}=0} \equiv \\
& f_{\rho} m_{\rho} p_{\nu} \frac{\varepsilon^{(\lambda)} \cdot z}{p \cdot z} \int_{0}^{1} d u e^{i(u-\bar{u}) p \cdot z} \phi_{\rho}^{\|}(u, \mu),
\end{aligned}
$$

where

$$
\mathcal{P}(z,-z)=\mathcal{P} \exp \left(-i g \int_{-z}^{z} d w^{\mu} A_{\mu}(w)\right)
$$

is the path-ordered exponential needed to maintain gauge invariance, $\mu$ is a renormalisation scale, $u$ is the momentum fraction of a quark, $\bar{u}=1-u$ and $\varepsilon^{(\lambda)}$ is the polarisation vector for a vector meson with polarisation state $\lambda$. The distribution amplitudes are normalised by

$$
\int_{0}^{1} d u \phi(u, \mu)=1 .
$$

The definitions above involve the pion and rho-meson decay constants defined by

$$
\begin{aligned}
& \left\langle 0\left|\bar{q}_{2} \gamma_{\mu} \gamma_{5} q_{1}\right| \pi(p)\right\rangle=i f_{\pi} p_{\mu}, \\
& \left\langle 0\left|\bar{q}_{2} \gamma_{\mu} q_{1}\right| \rho(p ; \lambda)\right\rangle=f_{\rho} m_{\rho} \varepsilon_{\mu}^{(\lambda)}
\end{aligned}
$$

The vector meson decay constant, $f_{\rho}$, and its coupling to the tensor current, $f_{\rho}^{T}$, are of interest in their own right and we have previously calculated [13] the ratios $f_{V}^{T} / f_{V}$, for $V \in\left\{\rho, K^{*}, \phi\right\}$ as part of our domain-wall fermion (DWF) phenomenology programme.

\section{B. Moments}

Moments of light-cone DAs are defined by:

$$
\left\langle\xi^{n}\right\rangle_{\pi}(\mu)=\int_{0}^{1} d u \xi^{n} \phi(u, \mu)
$$

where $\xi \equiv u-\bar{u}=2 u-1$ is the difference between the longitudinal momentum fractions.

Since the moments are obtained from matrix elements of local operators [14] we can study them using lattice QCD. The light-cone matrix elements which define the DAs themselves are not amenable to standard lattice techniques, since in Euclidean space the light-cone has been rotated to the complex direction. By expanding the non-local operators on the light cone, we obtain symmetric, traceless twist-2 operators. With the following conventions for continuum covariant derivatives,

$$
\vec{D}_{\mu}=\vec{\partial}_{\mu}+i g A_{\mu}, \quad \overleftarrow{D}_{\mu}=\overleftarrow{\partial}_{\mu}-i g A_{\mu}, \quad \overleftrightarrow{D}_{\mu}=\overleftarrow{D}_{\mu}-\vec{D}_{\mu}
$$

the expressions relating the moments of DAs to the corresponding local matrix elements are:

$$
\begin{aligned}
\left\langle 0\left|\bar{q}(0) \gamma_{\rho} \gamma_{5} \stackrel{\leftrightarrow}{D}_{\mu} s(0)\right| K(p)\right\rangle & =\left\langle\xi^{1}\right\rangle_{K} f_{K} p_{\rho} p_{\mu} \\
\left\langle 0\left|\bar{q}(0) \gamma_{\rho} \gamma_{5} \stackrel{\leftrightarrow}{D}_{\mu} \stackrel{\leftrightarrow}{D}_{\nu} q(0)\right| \pi(p)\right\rangle & =-i\left\langle\xi^{2}\right\rangle_{\pi} f_{\pi} p_{\rho} p_{\mu} p_{\nu} \\
\left\langle 0\left|\bar{q}(0) \gamma_{\rho} \stackrel{\leftrightarrow}{D}_{\mu} s(0)\right| K^{*}(p, \lambda)\right\rangle & =\left\langle\xi^{1}\right\rangle_{K^{*}}^{\|} f_{K^{*}} m_{K^{*}} \frac{1}{2}\left(p_{\mu} \varepsilon_{\nu}^{(\lambda)}+p_{\nu} \varepsilon_{\mu}^{(\lambda)}\right) \\
\left\langle 0\left|\bar{q}(0) \gamma_{\rho} \stackrel{\leftrightarrow}{D}_{\mu} \stackrel{\leftrightarrow}{D}_{\nu} q(0)\right| \rho(p, \lambda)\right\rangle & =-i\left\langle\xi^{2}\right\rangle_{\rho}^{\|} f_{\rho} m_{\rho} \frac{1}{3}\left(\varepsilon_{\rho}^{(\lambda)} p_{\mu} p_{\nu}+\varepsilon_{\mu}^{(\lambda)} p_{\nu} p_{\rho}+\varepsilon_{\nu}^{(\lambda)} p_{\rho} p_{\mu}\right)
\end{aligned}
$$


The operators in the matrix elements above are all to be considered symmetric and traceless in the free Lorentz indices. Meson-meson rather than meson-vacuum matrix elements of the same operators lead to moments of generalised parton distributions (GPDs).

Recent analyses, especially those based on QCD sum rules, deal instead with the Gegenbauer moments, which arise from a conformal expansion [15, 16], in which the conformal invariance of (classical) massless QCD is used to separate longitudinal and transverse degrees of freedom, analogous to the partial wave expansion in ordinary quantum mechanics. All dependence on the longitudinal momentum fractions is described by orthogonal polynomials that form an irreducible representation of the collinear subgroup of the conformal group, $\mathrm{SL}(2, \mathbb{R})$. The transverse-momentum dependence is represented as the scale-dependence of the relevant operators and is governed by renormalisation-group equations. The different 'partial waves', labelled by different conformal spins, do mix but not to leading-logarithmic accuracy. Conformal spin is thus a good quantum number in hard processes up to small corrections of order $\alpha_{s}^{2}$.

The asymptotic $Q^{2} \rightarrow \infty \mathrm{DA}$ is known from perturbative QCD: $\phi_{\mathrm{as}}=6 u \bar{u}$. For the leading-twist quarkantiquark DAs that we are interested in, the conformal expansion can then be conveniently written as:

$$
\phi(u, \mu)=6 u \bar{u}\left(1+\sum_{n=1}^{\infty} a_{n}(\mu) C_{n}^{3 / 2}(2 u-1)\right)
$$

where $C_{n}^{3 / 2}$ are Gegenbauer polynomials. To one-loop order the Gegenbauer moments, $a_{n}$, renormalise multiplicatively [16]:

$$
a_{n}(\mu)=a_{n}\left(\mu_{0}\right)\left(\frac{\alpha_{s}(\mu)}{\alpha_{s}\left(\mu_{0}\right)}\right)^{\left(\gamma_{(n)}-\gamma_{(0)}\right) / \beta_{0}}
$$

The one-loop anomalous dimensions are:

$$
\gamma_{(n)}=\gamma_{(n)}^{\|}=C_{F}\left(1-\frac{2}{(n+1)(n+2)}+4 \sum_{j=2}^{n+1} 1 / j\right) \text {, }
$$

where $C_{F}=4 / 3$. Since the moments are positive and increase with $n$, the effects of higher-order Gegenbauer polynomials are damped at higher scales as the DAs approach their asymptotic form. The conformal expansion can thus be truncated. Quantities such as the pion's electromagnetic form factor, for example, are given by convolutions in which the kernels are slowly-varying and the strongly-oscillating Gegenbauer polynomials are washed out. The same conclusion is reached by considering, rather than the conformal expansion, the diagonalisation of the ERBL equations [1, 4, 17, 18] which govern the evolution of the DAs much as the DGLAP equations 19 22 govern the evolution of PDFs.

We can obtain values for the Gegenbauer moments from lattice simulations since the Gegenbauer moments are combinations of ordinary moments of equal and lower order, e.g.:

$$
a_{1}=\frac{5}{3}\left\langle\xi^{1}\right\rangle, \quad a_{2}=\frac{7}{12}\left(5\left\langle\xi^{2}\right\rangle-1\right) .
$$

\section{Status}

In this section, we summarise what is currently known about leading-twist light-meson distribution amplitudes. For mesons of definite G-parity, there is a symmetry under the interchange $u \leftrightarrow \bar{u}$ of the two momentum fractions. In these cases, the distribution amplitude is an even function of $\xi=u-\bar{u}$ and the odd moments therefore vanish. Thus, $\left\langle\xi^{1}\right\rangle_{\pi},\left\langle\xi^{1}\right\rangle_{\rho}^{\|}$and $\left\langle\xi^{1}\right\rangle_{\phi}^{\|}$all vanish, while $\left\langle\xi^{1}\right\rangle_{K}$ and $\left\langle\xi^{1}\right\rangle_{K^{*}}^{\|}$are SU(3)-flavour breaking effects.

Since $\left\langle\xi^{1}\right\rangle_{K}$ is the average difference between the fractions of longitudinal momentum carried by the strange and light quarks,

$$
\left\langle\xi^{1}\right\rangle_{K}(\mu)=\int_{0}^{1} d u(2 u-1) \phi_{K}(u, \mu)=\langle 2 u-1\rangle,
$$

we may expect from the constituent quark model that the sign of $\left\langle\xi^{1}\right\rangle_{K}=\frac{3}{5} a_{1}^{K}$ is positive and this is indeed the case. In fact, $\left\langle\xi^{1}\right\rangle_{K}$ is an important $\mathrm{SU}(3)$-breaking parameter and is relevant for predictions of $B$-decay transitions such as $B \rightarrow K, K^{*}$ [23]. For example, a light-cone sum rule analysis leads to [24]:

$$
\frac{f_{+}^{B K}(0)}{f_{+}^{B \pi}(0)}=\frac{f_{K}}{f_{\pi}}\left(1+c_{1} a_{1}^{K}\right)+\ldots
$$

where $f_{+}^{B P}(0)$ is the vector $B \rightarrow P$ form factor at zero momentum transfer and $c_{1} \sim O(1)$. Other examples include the ratio of the weak radiative decay amplitudes $B \rightarrow \rho \gamma$ and $B \rightarrow K^{*} \gamma$, where the main theoretical error originates from such $\mathrm{SU}(3)$-breaking effects. The measured ratio of these decay rates allows for a determination of the ratio of CKM matrix elements $\left|V_{t s}\right| /\left|V_{t d}\right|$.

There have been three main approaches to the study of DAs: extraction from experimental data, calculations using QCD sum rules and lattice calculations. The overall normalisations are given by local hadronic matrix elements, essentially the decay constants, which have already been discussed and are partly accessible experimentally, and partly have to be calculated theoretically. The shapes of the leading-twist distribution amplitudes, in the form of the Gegenbauer moments, can be determined from experiments by analysing data on form factors such as $F_{\gamma \gamma^{*} \pi}$, which was studied by the CLEO experiment [25], and the pion's electromagnetic form factor, $F_{\pi}^{\mathrm{em}}[26]$. There is a lack of sufficiently accurate data, however, and it is difficult to avoid contamination from other hadronic uncertainties and higher twist effects. As a result, the existing experimental constraints are not very stringent. 
Moments of DAs, then, must largely be determined from theory. Lattice [23, 27 -32] and sum rule 33 36] studies have usually focussed on the second moment of the pion's distribution amplitude. However, the early lattice results were largely exploratory while sum rule results have an irreducible error of $\sim 20 \%$ because it is not possible properly to isolate the hadronic states.

The first moment of the kaon's distribution amplitude, for example, has in the past been determined mainly from QCD sum rules, and representative results include:

$$
a_{1}^{K}(1 \mathrm{GeV})= \begin{cases}0.05(2) & {[37]} \\ 0.10(12) & {[38]} \\ 0.050(25) & {[39]} \\ 0.06(3) & {[40]}\end{cases}
$$

These results all have the expected sign, but the uncertainties are around $50 \%$. The reduction of such uncertainties is the chief motivation of the lattice programme. In an earlier publication [41, 42], we obtained $\left\langle\xi^{1}\right\rangle_{K}(2 \mathrm{GeV}) \equiv 3 / 5 a_{K}^{1}(2 \mathrm{GeV})=0.032(3)$. We note that in addition to the UKQCD/RBC programme for the calculation of DA moments on the lattice using $N_{f}=2+1$ domain-wall fermions, there is a UKQCD/QCDSF programme using $N_{f}=2$ improved Wilson quarks [23]. QCDSF have also published results for moments of baryon DAs [28]. Lattice results for hadronic distribution amplitudes are considered in a recent review of hadron structure from lattice QCD in [43].

The plan for the remainder of this paper is as follows. In Sec. II] we discuss the extraction of bare moments of distribution amplitudes from Euclidean lattice correlation functions (we use 'bare' or 'latt' to denote quantities before matching from the lattice to the continuum). In Sec. III we give the details of our numerical calculations and present the bare results. The renormalisation of those bare results is described in Sec. IV] We then present our summary in Sec. V].

\section{BARE MOMENTS FROM LATTICE CORRELATION FUNCTIONS}

In this section, we describe our general strategy for the lattice calculation of the unrenormalised lowest moments of light meson distribution amplitudes. We obtain expressions for the first and second moments $\left\langle\xi^{1}\right\rangle$ and $\left\langle\xi^{2}\right\rangle$ for pseudoscalar mesons and for the longitudinal moments $\left\langle\xi^{1}\right\rangle^{\|}$and $\left\langle\xi^{2}\right\rangle^{\|}$for vector mesons, in terms of Euclidean lattice correlation functions which can be computed by Monte Carlo integration of the QCD path integral. In each case, we consider a generic meson having valence quark content $\bar{q}_{2} q_{1}$, where the subscripts indicate that the flavours of the two quarks may be different. We will see below that we can obtain all of these moments from ratios of two-point correlation functions and thus we expect to benefit from a significant reduction of the statistical fluctuations.

\section{A. Lattice Operators}

We now define the lattice operators used in the correlation functions from which we extract the moments of the distribution amplitudes. We use the following interpolating operators for the pseudoscalar and vector mesons:

$$
\begin{aligned}
P(x) & \equiv \bar{q}_{2}(x) \gamma_{5} q_{1}(x), \\
V_{\mu}(x) & \equiv \bar{q}_{2}(x) \gamma_{\mu} q_{1}(x), \\
A_{\mu}(x) & \equiv \bar{q}_{2}(x) \gamma_{\mu} \gamma_{5} q_{1}(x) .
\end{aligned}
$$

Although we have written $P, V$ and $A$ as local operators in Eq. (17), in the numerical simulations we use smeared operators at the source of our correlation functions in order to improve the overlap with the lightest meson states. Since the effects of smearing cancel in the ratios constructed below, the discussion in this section holds for both smeared and local interpolating operators. We explain the details of our smearing procedures in Sec. III A. The operators in Eqs. (9) from which the moments of the distribution amplitudes are obtained are of course local operators.

In constructing the lattice operators of Eqs. (9), we use the following symmetric left- and right-acting covariant derivatives:

$\vec{D}_{\mu} \psi(x)=\frac{1}{2 a}[U(x, x+\hat{\mu}) \psi(x+\hat{\mu})-U(x, x-\hat{\mu}) \psi(x-\hat{\mu})]$,

$\bar{\psi}(x) \overleftarrow{D}_{\mu}=\frac{1}{2 a}[\bar{\psi}(x+\hat{\mu}) U(x+\hat{\mu}, x)-\bar{\psi}(x-\hat{\mu}) U(x-\hat{\mu}, x)]$

where $U(x, y)$ is the gauge link going from site $x$ to site $y$ and $\hat{\mu}$ is a vector of length $a$ in the direction $\mu$ ( $a$ denotes the lattice spacing). The operators of interest are then defined by

$$
\begin{aligned}
\mathcal{O}_{\{\rho \mu\}}(x) & \equiv \bar{q}_{2}(x) \gamma_{\{\rho} \stackrel{\leftrightarrow}{D}_{\mu\}} q_{1}(x), \\
\mathcal{O}_{\{\rho \mu \nu\}}(x) & \equiv \bar{q}_{2}(x) \gamma_{\{\rho} \stackrel{\leftrightarrow}{D}_{\mu} \stackrel{\leftrightarrow}{D}_{\nu\}} q_{1}(x), \\
\mathcal{O}_{\{\rho \mu\}}^{5}(x) & \equiv \bar{q}_{2}(x) \gamma_{\{\rho} \gamma_{5} \stackrel{\leftrightarrow}{D}_{\mu\}} q_{1}(x), \\
\mathcal{O}_{\{\rho \mu \nu\}}^{5}(x) & \equiv \bar{q}_{2}(x) \gamma_{\{\rho} \gamma_{5} \stackrel{\leftrightarrow}{D}_{\mu} \stackrel{\leftrightarrow}{D}_{\nu\}} q_{1}(x),
\end{aligned}
$$

where the braces in the subscripts indicate symmetrisation of the enclosed Lorentz indices, $\left\{\mu_{1} \ldots \mu_{n}\right\} \equiv$ $\sum_{\text {perms } s}\left\{\mu_{s(1)} \ldots \mu_{s(n)}\right\} / n !$.

\section{B. Operator Mixing}

In the continuum the operators in Eq. (20) transform as second- or third-rank tensors under the Lorentz group. On the lattice however we must consider their transformation properties under the hypercubic group $\mathcal{H}_{4}$ of reflections and $\pi / 2$ rotations, together with the discrete symmetries parity $P$ and charge-conjugation $C$, where 
the possibilities for operator mixing are increased. A detailed study of the transformations of these operators under $\mathcal{H}_{4}$ has been performed in [44].

The choice of Lorentz indices in the operators used in simulations is important both to keep the operator mixing simple and also to enable the extraction of matrix elements using as few non-zero components of momentum as possible. The latter is to avoid the associated discretisation effects and statistical degradation. $\mathcal{O}_{\{\rho \mu\}}$ and $\mathcal{O}_{\{\rho \mu\}}^{5}$ renormalise multiplicatively under $\mathcal{H}_{4}$ when $\rho \neq \mu$. In the notation of [45], these operators transform under the 6 -dimensional $6^{(+)}\left(\right.$for $\left.\mathcal{O}_{\{\rho \mu\}}^{5}\right)$ or $6^{(-)}$ (for $\mathcal{O}_{\{\rho \mu\}}$ ) irreducible representations of $\mathcal{H}_{4}$. The choice $\mu \neq \rho$ is the most convenient one for the extraction of the first moment of the distribution amplitudes. Charge conjugation symmetry combined with $\mathcal{H}_{4}$ ensures that there is no mixing with operators containing total derivatives.

It is also possible to obtain the first moment from the four operators $\mathcal{O}_{\{\mu \mu\}}\left(\right.$ or $\left.\mathcal{O}_{\{\mu \mu\}}^{5}\right)$, which each transform as four-dimensional reducible representations containing a singlet. The three traceless operators transform as the 3dimensional irreducible representation $(3,1)^{(+)}$(without $\gamma^{5}$ ) or $(3,1)^{(-)}\left(\right.$with $\gamma^{5}$ ). Subtracting the trace involves the subtraction of a power divergence, so for the first moment of the distribution amplitude of the $K$ and $K^{*}$ we avoid this by evaluating the matrix elements of $\mathcal{O}_{\{\rho \mu\}}^{5}$ and $\mathcal{O}_{\{\rho \mu\}}$ respectively with $\rho \neq \mu$.

Similarly for the second moment of the distribution amplitudes the most convenient choice is to use $\mathcal{O}_{\{\rho \mu \nu\}}^{5}$ or $\mathcal{O}_{\{\rho \mu \nu\}}$ with all three indices different, which transform as the $(\overline{1 / 2,1 / 2})^{(+)}$and $(\overline{1 / 2,1 / 2})^{(-)}$4-dimensional irreducible representations respectively. Charge conjuga- tion symmetry allows mixing of $\mathcal{O}_{\{\rho \mu \nu\}}^{5}$ and $\mathcal{O}_{\{\rho \mu \nu\}}$ with operators containing total derivatives:

$$
\begin{aligned}
& \mathcal{O}_{\{\rho \mu \nu\}}^{5}(x) \text { mixes with } \partial_{\{\rho} \partial_{\mu}\left(\bar{q}_{2}(x) \gamma_{\nu\}} \gamma_{5} q_{1}(x)\right) \\
& \mathcal{O}_{\{\rho \mu \nu\}}(x) \text { mixes with } \partial_{\{\rho} \partial_{\mu}\left(\bar{q}_{2}(x) \gamma_{\nu\}} q_{1}(x)\right) .
\end{aligned}
$$

The moments of the distribution functions are obtained from non-forward matrix elements between a meson at non-zero four momentum and the vacuum, so the totalderivative operators must be included in the analysis.

\section{C. $\left\langle\xi^{1}\right\rangle_{P}$ and $\left\langle\xi^{2}\right\rangle_{P}$ from Correlation Function Ratios}

To obtain the first and second moments of the pseudoscalar meson distribution amplitude, $\left\langle\xi^{1}\right\rangle$ and $\left\langle\xi^{2}\right\rangle$, we consider the following two-point correlation functions:

$$
\begin{aligned}
C_{A_{\nu} P}(t, \boldsymbol{p}) & \equiv \sum_{\boldsymbol{x}} e^{i \boldsymbol{p} \cdot \boldsymbol{x}}\left\langle 0\left|A_{\nu}(t, \boldsymbol{x}) P^{\dagger}(0)\right| 0\right\rangle, \\
C_{\{\rho \mu\}}^{5}(t, \boldsymbol{p}) & \equiv \sum_{\boldsymbol{x}} e^{i \boldsymbol{p} \cdot \boldsymbol{x}}\left\langle 0\left|\mathcal{O}_{\{\rho \mu\}}^{5}(t, \boldsymbol{x}) P^{\dagger}(0)\right| 0\right\rangle, \\
C_{\{\rho \mu \nu\}}^{5}(t, \boldsymbol{p}) & \equiv \sum_{\boldsymbol{x}} e^{i \boldsymbol{p} \cdot \boldsymbol{x}}\left\langle 0\left|\mathcal{O}_{\{\rho \mu \nu\}}^{5}(t, \boldsymbol{x}) P^{\dagger}(0)\right| 0\right\rangle .
\end{aligned}
$$

For a generic pseudoscalar meson $P$, we define $Z_{P} \equiv\left\langle P(p)\left|P^{\dagger}\right| 0\right\rangle$ and the bare decay constant by $\left\langle 0\left|A_{\nu}\right| P(p)\right\rangle \equiv i p_{\nu} f_{P}^{\text {bare }}$. The operators $P^{\dagger}(0)$ in Eqs. (21) are smeared as explained below. At large Euclidean times $t$ and $T-t$, the correlation functions defined above tend towards:

$$
\begin{aligned}
C_{A_{\nu} P}(t, \boldsymbol{p}) & \rightarrow \frac{Z_{P} f_{P}^{\text {bare }} e^{-E_{P} T / 2} \sinh \left((t-T / 2) E_{P}\right)}{E_{P}} i p_{\nu}, \\
C_{\{\rho \mu\}}^{5}(t, \boldsymbol{p}) & \rightarrow \frac{Z_{P} f_{P}^{\text {bare }} e^{-E_{P} T / 2} \sinh \left((t-T / 2) E_{P}\right)}{E_{P}} i p_{\rho} i p_{\mu}\left\langle\xi^{1}\right\rangle^{\text {bare }}, \\
C_{\{\rho \mu \nu\}}^{5}(t, \boldsymbol{p}) & \rightarrow \frac{Z_{P} f_{P}^{\text {bare }} e^{-E_{P} T / 2} \sinh \left((t-T / 2) E_{P}\right)}{E_{P}} i p_{\rho} i p_{\mu} i p_{\nu}\left\langle\xi^{2}\right\rangle^{\text {bare }} .
\end{aligned}
$$

We can extract bare values for the first and second moments of the pseudoscalar meson distribution amplitudes from the following ratios of correlation functions:

$$
\begin{aligned}
R_{\{\rho \mu\} ; \nu}^{P}(t, \boldsymbol{p}) & \equiv \frac{C_{\{\rho \mu\}}^{5}(t, \boldsymbol{p})}{C_{A_{\nu} P}(t, \boldsymbol{p})} \rightarrow i \frac{p_{\rho} p_{\mu}}{p_{\nu}}\left\langle\xi^{1}\right\rangle^{\text {bare }}, \\
R_{\{\rho \mu \nu\} ; \sigma}^{P}(t, \boldsymbol{p}) & \equiv \frac{C_{\{\rho \mu \nu\}}^{5}(t, \boldsymbol{p})}{C_{A_{\sigma} P}(t, \boldsymbol{p})} \rightarrow-\frac{p_{\rho} p_{\mu} p_{\nu}}{p_{\sigma}}\left\langle\xi^{2}\right\rangle^{\text {bare }} .
\end{aligned}
$$

Keeping in mind the operator mixing outlined above, we obtain the first moment from $R_{\{\rho 4\} ; 4}^{P}(t, \boldsymbol{p})$ (the index 4 corresponds to the time direction) with $\rho=1,2$ or 3 and a single non-zero component of momentum, $\left|p_{\rho}\right|=2 \pi / L$. The second moment is extracted from $R_{\{\rho \mu 4\} ; 4}^{P}(t, \boldsymbol{p})$ with at least two non-zero components of momentum. We take $\rho, \mu=1,2$ or 3 with $\rho \neq \mu$ and $\left|p_{\rho}\right|=\left|p_{\mu}\right|=2 \pi / L$. We present more details in Sec. IIIB

Apart from isolating the moments of the DAs as much as possible by cancelling $Z_{P}, f_{P}^{\text {bare }}$ and most of the energy dependence from Eqs. (23) and (24), the ratios also simplify the effect of mixing with total derivative operators. These operators have matrix elements proportional to (22) with which we build a ratio similar to (25b). Hence 
the contribution of the mixing term becomes trivial and does not have to be computed explicitly. It enters as an additive constant when renormalising the bare moments as we will discuss later.

\section{D. $\left\langle\xi^{1}\right\rangle_{V}^{\|}$and $\left\langle\xi^{2}\right\rangle_{V}^{\|}$from Correlation Function Ratios}

The treatment of the vector meson's longitudinal distribution amplitude is analogous. We consider the following two-point correlation functions:

$$
\begin{aligned}
C_{V_{\mu} V_{\nu}}(t, \boldsymbol{p}) & \equiv \sum_{\boldsymbol{x}} e^{i \boldsymbol{p} \cdot \boldsymbol{x}}\left\langle 0\left|V_{\mu}(t, \boldsymbol{x}) V_{\nu}^{\dagger}(0)\right| 0\right\rangle, \\
C_{\{\rho \mu\} \nu}(t, \boldsymbol{p}) & \equiv \sum_{\boldsymbol{x}} e^{i \boldsymbol{p} \cdot \boldsymbol{x}}\left\langle 0\left|\mathcal{O}_{\{\rho \mu\}}(t, \boldsymbol{x}) V_{\nu}^{\dagger}(0)\right| 0\right\rangle, \\
C_{\{\rho \mu \nu\} \sigma}(t, \boldsymbol{p}) & \equiv \sum_{\boldsymbol{x}} e^{i \boldsymbol{p} \cdot \boldsymbol{x}}\left\langle 0\left|\mathcal{O}_{\{\rho \mu \nu\}}(t, \boldsymbol{x}) V_{\sigma}^{\dagger}(0)\right| 0\right\rangle .
\end{aligned}
$$

Again, the source operators $V^{\dagger}(0)$ are smeared. We define the bare longitudinal decay constant of a vector meson $V$, with polarisation index $\lambda$ and polarisation vector $\varepsilon_{\mu}^{(\lambda)}$, by $\left\langle 0\left|V_{\mu}\right| V(p, \lambda)\right\rangle \equiv f_{V}^{\text {bare }} m_{V} \varepsilon_{\mu}^{(\lambda)}$. Then, at large Euclidean times $t$ and $T-t$, the correlation functions defined above may be written:

$$
\begin{aligned}
C_{V_{\mu} V_{\nu}}(t, \boldsymbol{p}) & \rightarrow \frac{-\left(f_{V}^{\mathrm{bare}} m_{V}\right)^{2} e^{-E_{V} T / 2} \cosh \left((t-T / 2) E_{V}\right)}{E_{V}}\left(-g_{\mu \nu}+\frac{p_{\mu} p_{\nu}}{m_{V}^{2}}\right), \\
C_{\{\rho \mu\} \nu}(t, \boldsymbol{p}) & \rightarrow \frac{-i\left(f_{V}^{\mathrm{bare}} m_{V}\right)^{2} e^{-E_{V} T / 2}\left\langle\xi^{1}\right\rangle^{\| \text {bare }} \sinh \left((t-T / 2) E_{V}\right)}{E_{V}} \frac{1}{2}\left(-g_{\rho \nu} p_{\mu}-g_{\mu \nu} p_{\rho}+\frac{2 p_{\rho} p_{\mu} p_{\nu}}{\left.m_{V}^{2}\right),}\right. \\
C_{\{\rho \mu \nu\} \sigma}(t, \boldsymbol{p}) & \rightarrow \frac{\left(f_{V}^{\mathrm{bare}} m_{V}\right)^{2} e^{-E_{V} T / 2}\left\langle\xi^{2}\right\rangle^{\| \text {bare }} \sinh \left((t-T / 2) E_{V}\right)}{E_{V}} \frac{1}{3}\left(-g_{\rho \sigma} p_{\mu} p_{\nu}-g_{\mu \sigma} p_{\rho} p_{\nu}-g_{\nu \sigma} p_{\rho} p_{\mu}+\frac{3 p_{\rho} p_{\mu} p_{\nu} p_{\sigma}}{m_{V}^{2}}\right),
\end{aligned}
$$

where we have used the completeness relation for the polarisation vectors of massive vector particles, $\sum_{\lambda} \varepsilon_{\mu}^{(\lambda)} \varepsilon_{\nu}^{*(\lambda)}=$ $-g_{\mu \nu}+p_{\mu} p_{\nu} / m_{V}^{2}$. We extract bare values for the first and second moments from the following ratios:

$$
\begin{aligned}
& R_{\{\rho \mu\} \nu}^{V}(t, \boldsymbol{p}) \equiv \frac{C_{\{\rho \mu\} \nu}(t, \boldsymbol{p})}{\frac{1}{3} \sum_{i} C_{V_{i} V_{i}}(t, \boldsymbol{p}=0)} \rightarrow-i\left\langle\xi^{1}\right\rangle^{\| \text {bare }} \tanh \left((t-T / 2) E_{V}\right) \frac{1}{2}\left(-g_{\rho \nu} p_{\mu}-g_{\mu \nu} p_{\rho}+\frac{2 p_{\rho} p_{\mu} p_{\nu}}{m_{V}^{2}}\right) \\
& R_{\{\rho \mu \nu\} \sigma}^{V}(t, \boldsymbol{p}) \equiv \frac{C_{\{\rho \mu \nu\} \sigma}(t, \boldsymbol{p})}{\frac{1}{3} \sum_{i} C_{V_{i} V_{i}}\left(t, p_{i}=0,|\boldsymbol{p}|=\frac{2 \pi}{L}\right)}
\end{aligned}
$$

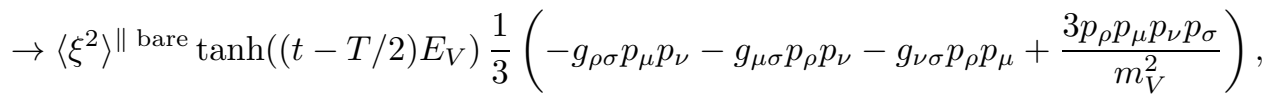

where the index $i$ runs over spatial dimensions only. We obtain the first moment from $R_{\{\rho 4\}}^{V}(t, \boldsymbol{p})$ at $\boldsymbol{p}=0$ by taking $\rho=\nu=1,2$ or 3 . The second moment is obtained from $R_{\{\rho \mu \nu\} \sigma}^{V}(t, \boldsymbol{p})$ by taking, for example, $\nu=4, \rho=$ $1, \mu=\sigma=2$ and a single non-zero component of $\boldsymbol{p}$ in the 1-direction.

\section{NUMERICAL SIMULATIONS AND RESULTS}

\section{A. Simulation Details}

Our numerical calculations are based upon gauge field configurations drawn from the joint datasets used for the broader UKQCD/RBC domain-wall fermion phenomenology programme. Configurations were generated with $N_{f}=2+1$ flavours of dynamical domainwall fermions and with the Iwasaki gauge action, using the Rational Hybrid Monte Carlo (RHMC) 46] 
algorithm on QCDOC computers [47-49] running the Columbia Physics System (CPS) software [50] and the BAGEL [51, 52] assembler generator.

Our set of gauge configurations includes data with two different volumes but at a single lattice spacing, thus giving us some indication of the size of finite volume effects but no ability to perform a continuum extrapolation. We therefore have an unavoidable systematic error which is, however, formally of $O\left(a^{2} \Lambda_{\mathrm{QCD}}^{2}\right)$ due to the automatic $O(a)$-improvement of the DWF action and operators. In the future, this limitation will be overcome by performing the analysis with a dataset with a finer lattice spacing with the same action (such a dataset is now available and is currently being calibrated). In the meantime, following the UKQCD/RBC procedure for these configurations [13], we ascribe a $4 \%$ uncertainty as the discretisation error on the moments. For both volumes, we have a single dynamical strange quark mass, close to its physical value. We use several independent ensembles with different light-quark masses $\left(m_{u}=m_{d}\right)$, all heavier than those found in nature. The hadronic spectrum and other properties of these configurations have been studied in detail and the results have been presented in 53] (for the lattice volume $\left.(L / a)^{3} \times T / a=16^{3} \times 32\right)$ and 13 (for the lattice volume $\left.24^{3} \times 64\right)$. In both cases the length of the fifth dimension is $L_{s}=16$.

The choice of bare parameters in our simulations is $\beta=2.13$ for the bare gauge coupling, $a m_{s}=0.04$ for the strange quark mass and $a m_{q}=0.03,0.02,0.01$ and, in the $24^{3}$ case only, 0.005 for the bare light-quark masses. A posteriori, the strange quark mass is found to be about $15 \%$ larger than its physical value. The lattice spacing is found to be $a^{-1}=1.729(28) \mathrm{GeV}[13$, giving physical volumes of $(1.83 \mathrm{fm})^{3}$ and $(2.74 \mathrm{fm})^{3}$. The lattice spacing and physical quark masses were obtained using the masses of the $\pi$ and $K$ pseudoscalar mesons and the triply-strange $\Omega$ baryon. The quark masses obtained in the $24^{3}$ study are shown in Table I] Owing to the remnant chiral symmetry breaking, the quark mass has to be corrected additively by the residual mass in the chiral limit, $a m_{\text {res }}=0.00315(2)$ [13]. The physical pion masses are as follows:

$$
m_{\pi} \simeq \begin{cases}670 \mathrm{MeV} & a m_{q}=0.03 \\ 555 \mathrm{MeV} & a m_{q}=0.02 \\ 415 \mathrm{MeV} & a m_{q}=0.01 \\ 330 \mathrm{MeV} & a m_{q}=0.005\end{cases}
$$

Measurements were performed using the UKhadron software package that makes use of both the BAGEL DWF inverter [51, 52 and elements of the SciDAC software library stack including the Chroma LQCD library [54] and QDP ++ . The details are summarised in Tables [II and III] We restrict our analysis to the unitary data for which the valence and sea quark masses are the same (partially-quenched data was used extensively in the studies of the chiral behaviour of the spectrum and decay constants in [13]). On the $16^{3}$ lattice, our dataset differs from that used in 53] in that the Markov chains have been extended for the heaviest light quark mass to give additional statistics, using an improved algorithm that decorrelated topology rather more quickly.

In order to improve the statistical sampling of the correlation functions, on each configuration we have averaged the results obtained from either 2, 3 or 4 sources spaced out along a lattice diagonal. In the $16^{3}$ case, for example, the sources used are at the origin, at $(4,4,4,8)$, $(8,8,8,16)$ and $(12,12,12,24)$. Statistical errors for observables are estimated using single-elimination jackknife, with measurements made on the same configuration but at different source positions put in the same jackknife bin because of the correlations expected between them. In order to lessen the effect of autocorrelations, we follow the same blocking procedures as in 53. and [13]. In the $16^{3}$ case, the span of the measurements in each block covers 50 molecular dynamics time units. In the $24^{3}$ case, for the $m_{q} a=0.005$ and $a m_{q}=0.01$ ensembles, each jackknife bin contains measurements from every 80 molecular dynamics time units, while for the $a m_{q}=0.02$ and $a m_{q}=0.03$ ensembles each bin contains measurements from every 40 molecular dynamics time units in order to have a reasonable number of bins for the analysis.

We use source smearing to improve the overlap with the mesonic states, either gauge-fixed hydrogen $S$ wavefunction smearing [55] with radius $r=3.5$ in lattice units or gauge invariant Gaussian smearing [56] with radius $r=4$.

\section{B. Results}

In order to extract $\left\langle\xi^{1}\right\rangle_{K}$ from the ratio $R_{\{\rho \mu\} ; \nu}^{P}(t, \boldsymbol{p})$ defined in (25a), we need the two correlation functions to be measured at $|\boldsymbol{p}| \neq 0$. Since we expect hadronic observables with larger lattice momenta to have larger lattice artefacts and statistical errors, we restrict the choice of indices to $\rho=\nu=4$ and $\mu=1,2$ or 3 with $|\boldsymbol{p}|=2 \pi / L$ (i.e., $p_{\mu}= \pm 2 \pi / L$ with the remaining two components of $\boldsymbol{p}$ equal to 0$) \cdot\left\langle\xi^{1}\right\rangle_{K}^{\text {bare }}$ can then be obtained from the ratio at large times:

$$
R_{\{4 k\} ; 4}^{P}\left(t, p_{k}= \pm 2 \pi / L\right)= \pm i \frac{2 \pi}{L}\left\langle\xi^{1}\right\rangle^{\text {bare }},
$$

with $|\boldsymbol{p}|=2 \pi / L$ and $k=1,2,3$. The plots in Fig. 1 1 show our results for $\left\langle\xi^{1}\right\rangle_{K}^{\text {bare }}$ as a function of $t$ obtained from the ratio $R_{\{4 k\} ; 4}^{P}\left(t, p_{k}= \pm 2 \pi / L\right)$ for the four values of the light-quark mass, combining results at $t$ with those at $T-t-1$. The results have been averaged over the three values of $k$ and, in total, the 6 equivalent lattice momenta with $|\boldsymbol{p}|=2 \pi / L$.

To obtain $\left\langle\xi^{2}\right\rangle_{\pi, K}^{\text {bare }}$ from the ratio $R_{\{\rho \mu \nu\} ; \sigma}^{P}(t, \boldsymbol{p})$ defined in (25b) we need two non-zero components of momentum, 
TABLE I. Lattice scale and unrenormalised quark masses in lattice units, from the $24^{4}$ lattices $\left[13\right.$ ]. Note $\tilde{m}_{X} \equiv m_{X}+m_{\text {res }}$. Only the statistical errors are given here.

\begin{tabular}{c|cccccc}
\hline \hline$a^{-1}[\mathrm{GeV}]$ & $a[\mathrm{fm}]$ & $a m_{u d}$ & $a \widetilde{m}_{u d}$ & $a m_{s}$ & $a \widetilde{m}_{s}$ & $a \widetilde{m}_{u d}: a \widetilde{m}_{s}$ \\
\hline $1.729(28)$ & $0.1141(18)$ & $-0.001847(58)$ & $0.001300(58)$ & $0.0343(16)$ & $0.0375(16)$ & $1: 28.8(4)$ \\
\hline \hline
\end{tabular}
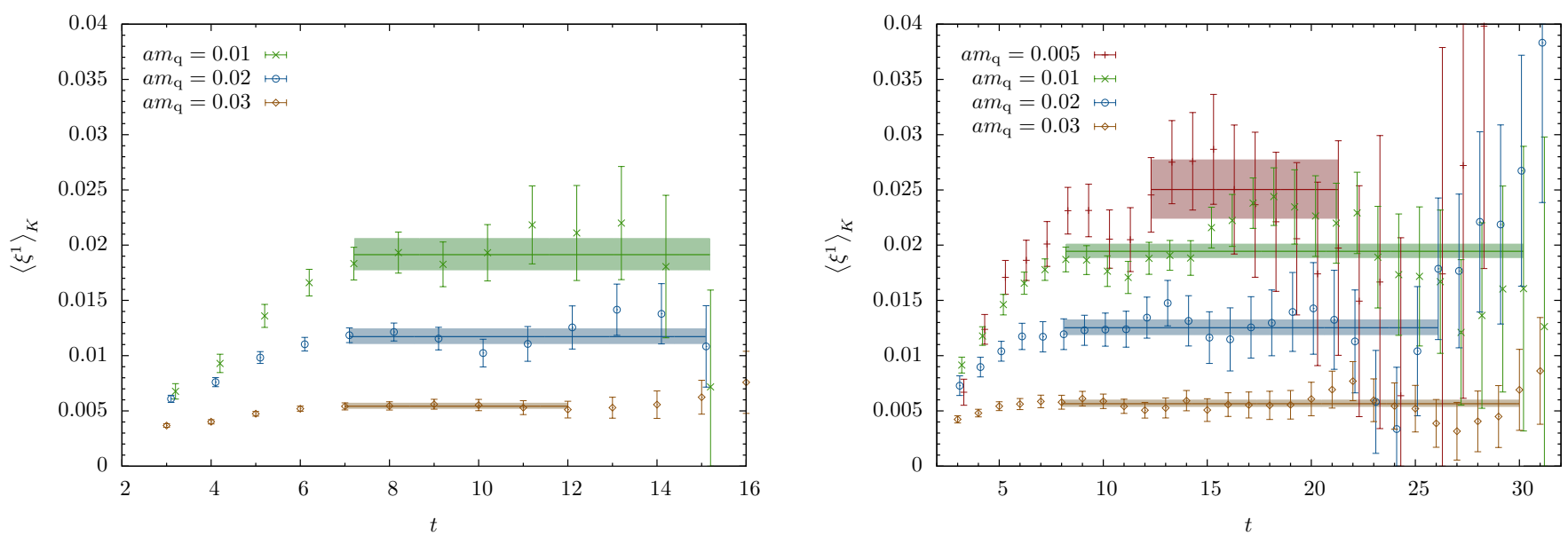

FIG. 1. Results for $\left\langle\xi^{1}\right\rangle_{K}^{\text {bare }}$ as a function of the time, on the $16^{3}$ (left) and $24^{3}$ (right) lattices. The shaded band shows the fit range, fitted value and its error.

TABLE II. Parameters for our $16^{3}$ dataset, which corresponds largely to that of [53]. The range and measurement separation $\Delta$ are specified in molecular dynamics time units. $N_{\text {meas }}$ is the number of measurements for each source position $t_{\mathrm{src}}$. The total number of measurements is therefore $N_{\text {meas }} \times N_{\text {src }}$, where $N_{\text {src }}$ is the number of different values for $t_{\text {src }}$. In the rightmost column, XY-XY denotes contraction of two quark propagators with $\mathrm{X}$-type smearing at source and $\mathrm{Y}$-type smearing at sink: $\mathrm{G}=$ Gaussian wavefunction, $\mathrm{L}=$ point.

\begin{tabular}{c|ccccc}
\hline \hline$m_{l}$ & Range & $\Delta$ & $N_{\text {meas }}$ & $t_{\text {src }}$ locations & Smearing \\
\hline 0.01 & $500-3990$ & 10 & 350 & $0,8,16,24$ & GL-GL \\
0.02 & $500-3990$ & 10 & 350 & $0,8,16,24$ & GL-GL \\
0.03 & $4030-7600$ & 10 & 358 & 0,16 & GL-GL \\
\hline \hline
\end{tabular}

TABLE III. Parameters for our $24^{3}$ dataset, which corresponds to the unitary part of the dataset of [13]. Columns as in Table $\amalg$ with addition of $\mathrm{H}=$ gauge-fixed hydrogen Swave smearing.

\begin{tabular}{c|ccccc}
\hline \hline$m_{l}$ & Range & $\Delta$ & $N_{\text {meas }}$ & $t_{\text {src }}$ locations & Smearing \\
\hline 0.005 & $900-4480$ & 20 & 180 & $0,32,16$ & HL-HL \\
0.01 & $800-3940$ & 10 & 315 & 0,32 & GL-GL \\
0.02 & $1800-3580$ & 20 & 90 & 0,32 & HL-HL \\
0.03 & $1260-3040$ & 20 & 90 & 0,32 & HL-HL \\
\hline \hline
\end{tabular}

so we use

$$
\begin{aligned}
R_{\{4 j k\} ; 4}^{P}\left(t, p_{j}= \pm 2 \pi / L,\right. & \left.p_{k}= \pm 2 \pi / L\right)= \\
& -\left( \pm \frac{2 \pi}{L}\right)\left( \pm \frac{2 \pi}{L}\right)\left\langle\xi^{2}\right\rangle^{\text {bare }}
\end{aligned}
$$

with $|\boldsymbol{p}|=\sqrt{2} 2 \pi / L, k, j=1,2,3$ and $k \neq j$. We average over all 4 momentum combinations appropriate to each of the 3 possible Lorentz index choices.

We may extract $\left\langle\xi^{1}\right\rangle_{K^{*}}^{\| \text {bare }}$ from the ratio $R_{\{\rho \mu\} \nu}^{V}(t, \boldsymbol{p})$ defined in (28a by considering only zero-momentum correlation functions. In the denominator, we average $C_{V_{i} V_{i}}(t, \boldsymbol{p}=0)$ over all 3 spatial directions. In the numerator, we average over $C_{\{41\} 1}(t, \boldsymbol{p}=0), C_{\{42\} 2}(t, \boldsymbol{p}=0)$ and $C_{\{43\} 3}(t, \boldsymbol{p}=0)$. Results are shown in Fig. 2 . $\left\langle\xi^{2}\right\rangle_{K^{*}, \rho, \phi}^{\| \text {bare }}$ is extracted from the ratio defined in (28b) by averaging $C_{V_{i} V_{i}}\left(t, p_{i}=0,|\boldsymbol{p}|=\frac{2 \pi}{L}\right)$ over all 4 appropriate momenta for all 3 spatial directions in the denominator. In the numerator we average over all possible combinations of $C_{\{4 i j\} i}\left(t, p_{j}= \pm \frac{2 \pi}{L},|\boldsymbol{p}|=\frac{2 \pi}{L}\right)$ with $i \neq j$. In principle we should include disconnected contributions in the $\phi$ correlation functions. We argue that these contributions are Zweig-suppressed however and can therefore be neglected.

If picking the fit range was not straightforward, we considered the correlation functions in the numerator and denominator separately. We identified and excluded from our fits the region where the excited states still contributed. We then chose the fit range aiming for a good $\chi^{2} /$ d.o.f. and a stable fit with respect to small variations of the lower bound of the range. Owing to the increasing noise when $t$ gets larger, the fits are insensitive to the upper bound of the fit range.

The $16^{3}$ and $24^{3}$ bare results are given in Tables IV]and V]respectively, complete with linear chiral extrapolations which, as we shall discuss in the next section, can be justified using chiral perturbation theory (at least in the 

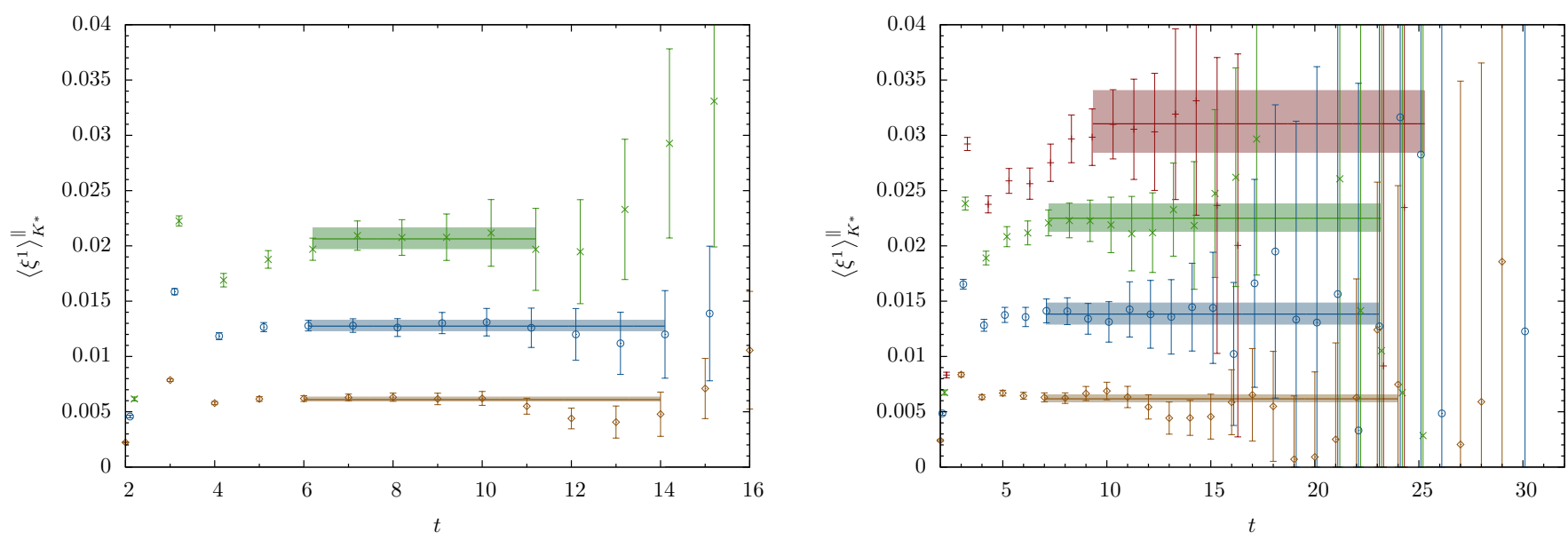

FIG. 2. Results for $\left\langle\xi^{1}\right\rangle_{K^{*}}^{\| \text {bare }}$ as a function of the time, on the $16^{3}$ (left) and $24^{3}$ (right) lattices. Symbols as in Fig. 1

pseudoscalar case).

\section{Quark Mass Extrapolations}

In leading-order chiral perturbation theory [57], $\left\langle\xi^{1}\right\rangle_{K}$ is proportional to $m_{s}-m_{u / d}$ without chiral logarithms:

$$
\left\langle\xi^{1}\right\rangle_{K}=\frac{8 B_{0}}{f^{2}}\left(m_{s}-m_{u / d}\right) b_{1,2}
$$

where $f$ and $B_{0}$ denote the usual chiral perturbation theory parameters and $b_{1,2}$ is a Wilson coefficient introduced in [57]. Our data shows clearly the effects of SU(3) symmetry breaking and is compatible with this expectation. We therefore perform a linear extrapolation in $a\left(m_{s}-m_{q}\right)$ to the physical point $a\left(m_{s}-m_{u d}\right)$, as shown in Fig. 3 . The second error quoted in the results in the chiral-limit for the first moments in Tables [IV] and $\nabla$ is due to the uncertainty in this physical point (determined using the quark masses in Table I). In this way we deal simultaneously with the usual light-quark mass extrapolation and with the strange quark mass extrapolation which is necessitated by our strange quark mass being approximately $15 \%$ too heavy. We have not constrained our fit to vanish in the $\mathrm{SU}(3)$ limit.

A similar linear behaviour is seen for $\left\langle\xi^{1}\right\rangle_{K^{*}}^{\| \text {bare }}$ (see Fig. 3), so we follow the same extrapolation procedure. We note a hint of a finite volume effect in the $K^{*}$ case but not in the $K$ case, which is contrary to what we would expect. Where we have $K^{*}$ results for both volumes at the same light-quark mass, however, they agree within the statistical uncertainties.

For the second moments, we also have some guidance from chiral perturbation theory [58]; there is no nonanalytic dependence at 1 loop and we should fit linearly in $m_{\pi}^{2}$. The dependence on the quark masses is very mild in any case and in fact our results for the $\rho, K^{*}$ and $\phi$ agree within the statistical errors. Therefore we perform a linear extrapolation in the light quark masses and neglect the effect of the too-heavy strange quark mass (see Fig. 4). We see no indication for finite size effects in the second moments when we compare the data points on the two different lattice volumes. They agree within their statistical errors.

\section{RENORMALISATION OF THE LATTICE COMPOSITE OPERATORS}

We now discuss the conversion of our bare lattice results to results in the $\overline{\mathrm{MS}}$ scheme. To reduce systematic uncertainties we have determined the renormalisation factors nonperturbatively in the $\mathrm{RI}^{\prime}$ / MOM scheme, continuing the work in [59], and convert to $\overline{\mathrm{MS}}$ using 3loop continuum perturbation theory [60, 61]. We begin, however, with a perturbative calculation of the renormalisation factors. The perturbative results have been used previously in [62, 63] and will provide a comparison to the nonperturbative results. The contribution to the second moment from mixing with a total-derivative operator is calculated perturbatively only. We will see that this contribution is small and is not accessible within the current nonperturbative scheme.

\section{A. Perturbative Renormalisation}

The perturbative matching from the lattice to $\overline{\mathrm{MS}}$ schemes is performed by comparing one-loop calculations of quark two-point one particle irreducible (1PI) functions with an insertion of the relevant bare lattice operator. This requires the evaluation of the diagrams shown in Fig. 5, together with wavefunction renormalisation factors, Fig. 6. For the first-moment operator, we define

$$
\mathcal{O}_{\{\rho \mu\}}^{\overline{\mathrm{MS}}}(\mu)=Z_{\mathcal{O}_{\{\rho \mu\}}}(\mu a) \mathcal{O}_{\{\rho \mu\}}^{\text {latt }}(a)
$$



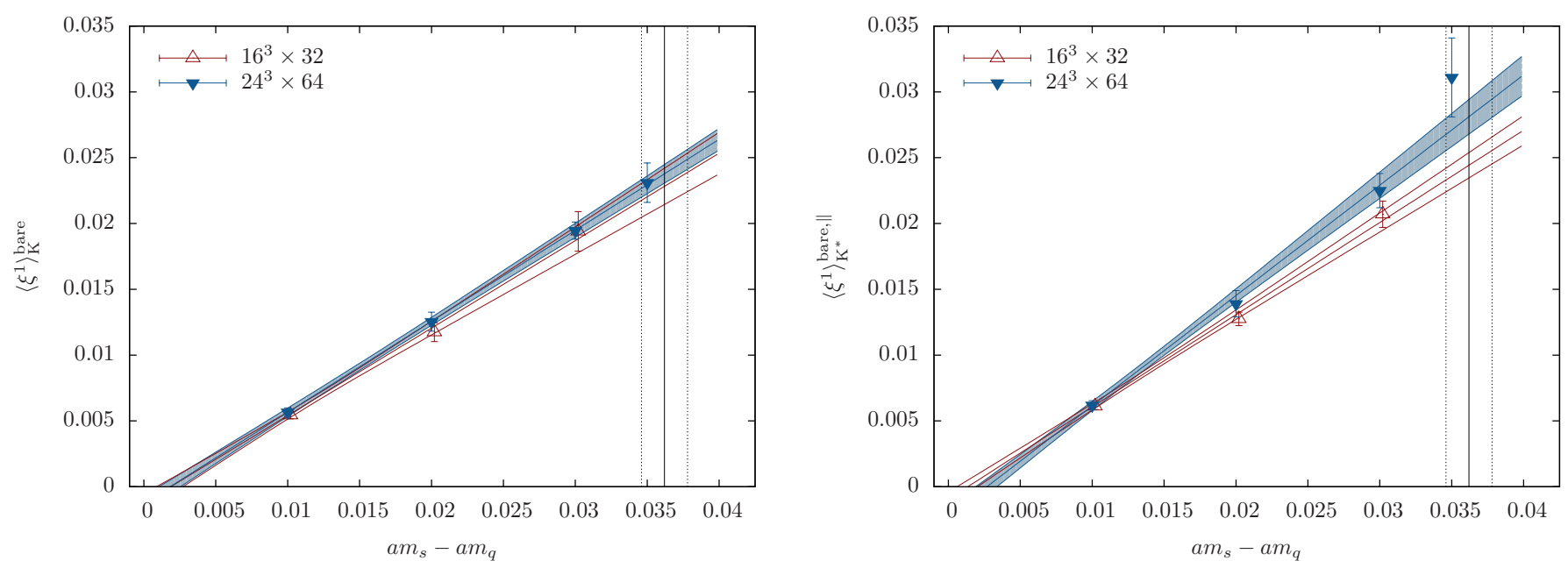

FIG. 3. Chiral extrapolations for $\left\langle\xi^{1}\right\rangle_{K}^{\text {bare }}$ and $\left\langle\xi^{1}\right\rangle_{K^{*}}^{\| \text {bare }}$. The extrapolation to the physical point is shown by the vertical solid line, with uncertainty, dominated by the uncertainty in the physical strange mass, indicated by the dotted lines.

TABLE IV. Summary of results for the bare values of the distribution amplitude moments on the $16^{3}$ lattices. The chiral extrapolations are discussed in Sec. [IIC] and the errors are statistical and (in the first moment case) due to the uncertainty in the physical point for the chiral extrapolation.

\begin{tabular}{l|lllll}
\hline \hline$a m_{u d}$ & 0.03 & 0.02 & 0.01 & 0.005 & $\chi$-limit \\
\hline$\left\langle\xi^{2}\right\rangle_{\pi}^{\text {bare }}$ & $0.110(2)$ & $0.109(2)$ & $0.113(4)$ & - & $0.112(5)$ \\
$\left\langle\xi^{1}\right\rangle_{K}^{\text {bare }}$ & $0.00543(27)$ & $0.01174(71)$ & $0.0194(15)$ & - & $0.0228(14)(11)$ \\
$\left\langle\xi^{2}\right\rangle_{K}^{\text {bare }}$ & $0.109(2)$ & $0.107(2)$ & $0.113(3)$ & - & $0.112(4)$ \\
$\left\langle\xi^{2}\right\rangle_{\rho}^{\| \text {bare }}$ & $0.113(4)$ & $0.100(5)$ & $0.116(6)$ & - & $0.109(10)$ \\
$\left\langle\xi^{1}\right\rangle_{K^{*}}^{\| \text {bare }}$ & $0.00610(24)$ & $0.01275(51)$ & $0.0207(10)$ & - & $0.02443(96)(107)$ \\
$\left\langle\xi^{2}\right\rangle_{K^{*}}^{\| \text {bare }}$ & $0.111(4)$ & $0.101(4)$ & $0.113(4)$ & - & $0.110(6)$ \\
$\left\langle\xi^{2}\right\rangle_{\phi}^{\| \text {bare }}$ & $0.109(3)$ & $0.100(3)$ & $0.109(3)$ & - & $0.107(5)$ \\
\hline \hline
\end{tabular}

For the second moment calculation we must take account of mixing with a total derivative operator (c.f. Sec. IIB). Adopting the notation

$$
\mathcal{O}_{D D}=\bar{\psi} \gamma_{\{\mu} \gamma_{5} \stackrel{\leftrightarrow}{D}_{\nu} \stackrel{\leftrightarrow}{D}_{\kappa\}} \psi, \quad \mathcal{O}_{\partial \partial}=\partial_{\{\nu} \partial_{\kappa} \bar{\psi} \gamma_{\mu\}} \gamma_{5} \psi
$$

with all Lorentz indices distinct and symmetrised, we need to determine

$$
\mathcal{O}_{D D}^{\overline{\mathrm{MS}}}(\mu)=Z_{D D, D D}(\mu a) \mathcal{O}_{D D}^{\mathrm{latt}}(a)+Z_{D D, \partial \partial}(\mu a) \mathcal{O}_{\partial \partial}^{\mathrm{latt}}(a) .
$$

The renormalisation factors are given by

$$
\begin{aligned}
Z_{\mathcal{O}_{\{\rho \mu\}}}(\mu a) & =\frac{1}{\left(1-w_{0}^{2}\right) Z_{w}}\left[1+\frac{\alpha C_{\mathrm{F}}}{4 \pi}\left(-\frac{16}{3} \ln (\mu a)+\Sigma_{1}^{\overline{\mathrm{MS}}}-\Sigma_{1}+V^{\overline{\mathrm{MS}}}-V\right)\right], \\
Z_{D D, D D}(\mu a) & =\frac{1}{\left(1-w_{0}^{2}\right) Z_{w}}\left[1+\frac{\alpha C_{\mathrm{F}}}{4 \pi}\left(-\frac{25}{3} \ln (\mu a)+\Sigma_{1}^{\overline{\mathrm{MS}}}-\Sigma_{1}+V_{D D}^{\overline{\mathrm{MS}}}-V_{D D}\right)\right], \\
Z_{D D, \partial \partial}(\mu a) & =\frac{1}{\left(1-w_{0}^{2}\right) Z_{w}} \frac{\alpha C_{\mathrm{F}}}{4 \pi}\left(\frac{5}{3} \ln (\mu a)+V_{\partial \partial}^{\overline{\mathrm{MS}}}-V_{\partial \partial}\right) .
\end{aligned}
$$

In the equations above $\left(1-w_{0}^{2}\right) Z_{w}$ is a characteristic normalisation factor for the physical quark fields in the domain-wall formalism. $Z_{w}$ represents an additive renormalisation of the large Dirac mass or domain-wall height $M=1-w_{0}$, which can be rewritten in multiplicative form at one-loop as

$$
Z_{w}=1+\frac{\alpha C_{\mathrm{F}}}{4 \pi} z_{w}, \quad z_{w}=\frac{2 w_{0}}{1-w_{0}^{2}} \Sigma_{w}
$$



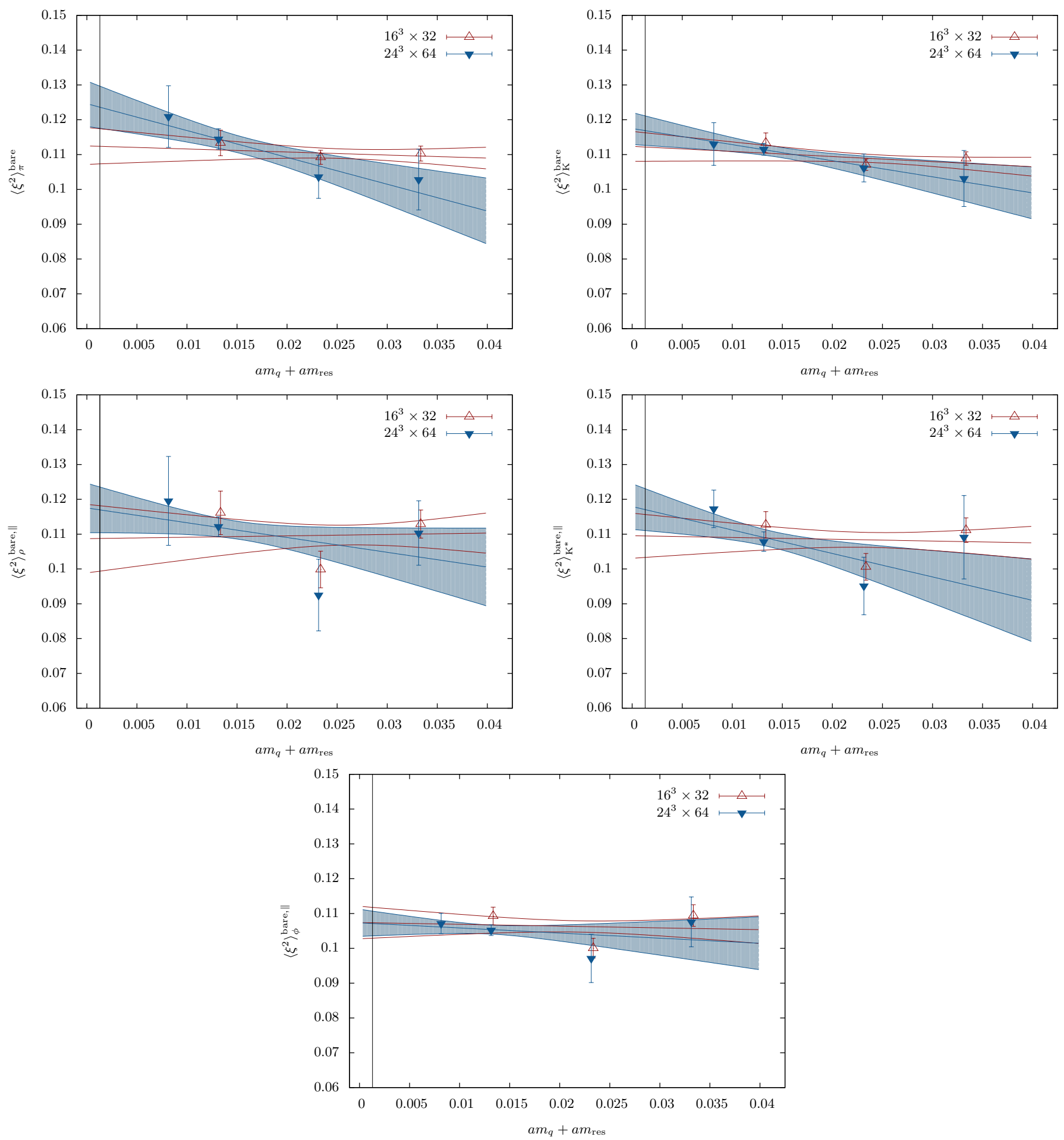

FIG. 4. Chiral extrapolations for $\left\langle\xi^{2}\right\rangle_{\pi}^{\text {bare }},\left\langle\xi^{2}\right\rangle_{K}^{\text {bare }},\left\langle\xi^{2}\right\rangle_{\rho}^{\| \text {bare }},\left\langle\xi^{2}\right\rangle_{K^{*}}^{\| \text {bare }}$ and $\left\langle\xi^{2}\right\rangle_{\phi}^{\| \text {bare }}$. The physical value for $a m_{q}+a m_{\text {res }}$ is shown by the solid vertical line in each case.

The one-loop correction $z_{w}$ becomes very large for certain choices of $M$ [64, 65], including that used in our numerical simulations, so that some form of mean-field improvement is necessary, as discussed below.

Terms with superscripts $\overline{\mathrm{MS}}$ in Eqs. (36), (37) and (38) arise from the continuum calculations, while unsuper- scripted terms come from the computations in the lattice scheme. To shorten some expressions below we will 
TABLE V. Summary of results for the bare values of the distribution amplitude moments on the $24^{3}$ lattices

\begin{tabular}{l|lllll}
\hline \hline$a m_{u d}$ & 0.03 & 0.02 & 0.01 & 0.005 & $\chi$-limit \\
\hline$\left\langle\xi^{2}\right\rangle_{\pi}^{\text {bare }}$ & $0.103(9)$ & $0.104(6)$ & $0.114(3)$ & $0.121(9)$ & $0.125(7)$ \\
$\left\langle\xi^{1}\right\rangle_{K}^{\text {bare }}$ & $0.00566(33)$ & $0.01254(72)$ & $0.01946(65)$ & $0.0231(15)$ & $0.02377(71)(110)$ \\
$\left\langle\xi^{2}\right\rangle_{K}^{\text {bare }}$ & $0.103(8)$ & $0.106(4)$ & $0.112(2)$ & $0.113(6)$ & $0.117(5)$ \\
$\left\langle\xi^{2}\right\rangle_{\rho}^{\| \text {bare }}$ & $0.110(9)$ & $0.093(10)$ & $0.112(3)$ & $0.120(13)$ & $0.118(7)$ \\
$\left\langle\xi^{1}\right\rangle_{K^{*}}^{\| \text {bare }}$ & $0.00619(35)$ & $0.0139(10)$ & $0.0225(13)$ & $0.0311(30)$ & $0.0281(13)(14)$ \\
$\left\langle\xi^{2}\right\rangle_{K^{*}}^{\| \text {bare }}$ & $0.109(12)$ & $0.095(8)$ & $0.108(3)$ & $0.117(5)$ & $0.118(7)$ \\
$\left\langle\xi^{2}\right\rangle_{\phi}^{\| \text {bare }}$ & $0.108(7)$ & $0.097(7)$ & $0.105(2)$ & $0.107(3)$ & $0.107(4)$ \\
\hline \hline
\end{tabular}

TABLE VI. Constants needed for the perturbative renormalisation of the first and second moment operators using domain-wall fermions and the Iwasaki gauge action $\left(c_{1}=-0.331\right) . \quad M$ is the domain-wall height, $c=\Sigma_{1}^{\overline{\mathrm{MS}}}-\Sigma_{1}+V^{\overline{\mathrm{MS}}}-V, c_{D D}=$ $\Sigma_{1}^{\overline{\mathrm{MS}}}-\Sigma_{1}+V_{D D}^{\overline{\mathrm{MS}}}-V_{D D}$ and $c_{\partial \partial}=V_{\partial \partial}^{\overline{\mathrm{MS}}}-V_{\partial \partial} . \Sigma_{1}, V, V_{D D}$ and $V_{\partial \partial}$ are dependent on the gauge and the infrared regulator: Feynman gauge and a gluon mass are used here. $V$ was calculated in [41], while $V_{D D}$ and $V_{\partial \partial}$ have been calculated as part of this work.

\begin{tabular}{|c|c|c|c|c|c|c|c|}
\hline$M$ & $\Sigma_{1}$ & $V$ & $c$ & $V_{D D}$ & $c_{D D}$ & $V_{\partial \partial}$ & $c_{\partial \partial}$ \\
\hline$\overline{0.1}$ & 4.6519 & -4.6297 & -0.9110 & -10.816 & 4.9838 & 0.5415 & $\overline{0.0279}$ \\
\hline 0.2 & 4.5193 & -4.5614 & -0.8468 & -10.698 & 4.9982 & 0.4285 & 0.1409 \\
\hline 0.3 & 4.4093 & -4.5101 & -0.7881 & -10.608 & 5.0179 & 0.3433 & 0.2262 \\
\hline 0.4 & 4.3158 & -4.4678 & -0.7369 & -10.533 & 5.0362 & 0.2729 & 0.2966 \\
\hline 0.5 & 4.2354 & -4.4311 & -0.6932 & -10.467 & 5.0509 & 0.2119 & 0.3575 \\
\hline 0.6 & 4.1665 & -4.3980 & -0.6574 & -10.407 & 5.0603 & 0.1573 & 0.4122 \\
\hline 0.7 & 4.1079 & -4.3673 & -0.6295 & -10.352 & 5.0639 & 0.1070 & 0.4625 \\
\hline 0.8 & 4.0593 & -4.3381 & -0.6101 & -10.300 & 5.0604 & 0.0597 & 0.5098 \\
\hline 0.9 & 4.0204 & -4.3097 & -0.5996 & -10.250 & 5.0489 & 0.0142 & 0.5552 \\
\hline 1. & 3.9915 & -4.2816 & -0.5988 & -10.200 & 5.0283 & -0.0303 & 0.5998 \\
\hline 1.1 & 3.9731 & -4.2529 & -0.6090 & -10.151 & 4.9970 & -0.0749 & 0.6443 \\
\hline 1.2 & 3.9664 & -4.2232 & -0.6321 & -10.100 & 4.9528 & -0.1205 & 0.6899 \\
\hline 1.3 & 3.9727 & -4.1916 & -0.6700 & -10.047 & 4.8933 & -0.1682 & 0.7376 \\
\hline 1.4 & 3.9943 & -4.1571 & -0.7261 & -9.9895 & 4.8147 & -0.2195 & 0.7889 \\
\hline 1.5 & 4.0343 & -4.1182 & -0.8050 & -9.9267 & 4.7119 & -0.2764 & 0.8458 \\
\hline 1.6 & 4.0974 & -4.0728 & -0.9135 & -9.8551 & 4.5771 & -0.3418 & 0.9112 \\
\hline 1.7 & 4.1905 & -4.0176 & -1.0618 & -9.7700 & 4.3989 & -0.4205 & 0.9899 \\
\hline 1.8 & 4.3249 & -3.9462 & -1.2676 & -9.6627 & 4.1572 & -0.5211 & 1.0905 \\
\hline 1.9 & 4.5209 & -3.8447 & -1.5651 & -9.5140 & 3.8125 & -0.6631 & 1.2325 \\
\hline
\end{tabular}
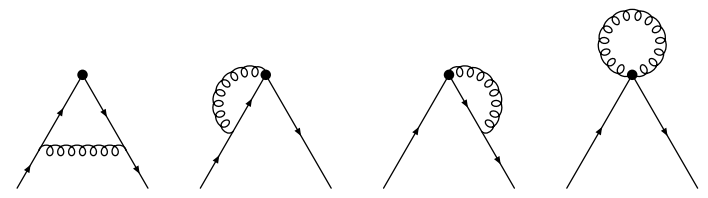

FIG. 5. One-loop vertex diagrams evaluated in the perturbative renormalisation of the 1 st and 2 nd moment operators.

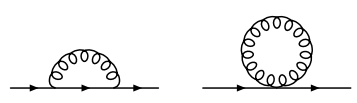

FIG. 6. One-loop diagrams for the quarks' wavefunction renormalisation. define

$$
\begin{aligned}
c & =\Sigma_{1}^{\overline{\mathrm{MS}}}-\Sigma_{1}+V^{\overline{\mathrm{MS}}}-V, \\
c_{D D} & =\Sigma_{1}^{\overline{\mathrm{MS}}}-\Sigma_{1}+V_{D D}^{\overline{\mathrm{MS}}}-V_{D D}, \\
c_{\partial \partial} & =V_{\partial \partial}^{\overline{\mathrm{MS}}}-V_{\partial \partial} .
\end{aligned}
$$

The terms $\Sigma_{1}^{\overline{\mathrm{MS}}}$ and $\Sigma_{1}$ come from quark wavefunction renormalisation, while $V^{\overline{\mathrm{MS}}}, V_{D D}^{\overline{\mathrm{MS}}}, V_{\partial \partial}^{\overline{\mathrm{MS}}}$ and $V, V_{D D}$,

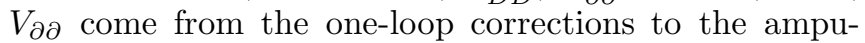
tated two-point function. They are given by "vertex" and "sail" diagrams, plus an operator tadpole diagram in the lattice case. $V_{D D}^{\overline{\mathrm{MS}}}$ and $V_{D D}$ can be isolated by computing the one-loop correction with equal incoming and outgoing quark momenta. Likewise $V_{\partial \partial}^{\overline{\mathrm{MS}}}$ and $V_{\partial \partial}$ are found by setting the incoming and outgoing quark momenta equal and opposite (the lattice tadpole diagram does not contribute in this case). Using naive dimensional regularisation (NDR) in Feynman gauge with a 
TABLE VII. Values for $z_{w}, z_{w}^{\mathrm{MF}}$ extracted from the results in [65], and $d_{f}$ extracted from [71].

\begin{tabular}{cccc}
\hline \hline$M$ & $z_{w}$ & $z_{w}^{\mathrm{MF}}$ & $d_{f}$ \\
\hline 0.1 & -243.86 & -86.579 & -0.02303 \\
0.2 & -113.29 & -39.501 & -0.01798 \\
0.3 & -69.404 & -23.830 & -0.01497 \\
0.4 & -47.077 & -15.949 & -0.01274 \\
0.5 & -33.278 & -11.142 & -0.01090 \\
0.6 & -23.648 & -7.8365 & -0.009315 \\
0.7 & -16.300 & -5.3538 & -0.007896 \\
0.8 & -10.263 & -3.3459 & -0.006589 \\
0.9 & -4.9617 & -1.6078 & -0.005379 \\
1.0 & 0.0 & 0.0 & -0.004261 \\
1.1 & 4.9442 & 1.5902 & -0.003227 \\
1.2 & 10.192 & 3.2748 & -0.002290 \\
1.3 & 16.136 & 5.1900 & -0.001485 \\
1.4 & 23.346 & 7.5350 & -0.0008650 \\
1.5 & 32.784 & 10.648 & -0.0005360 \\
1.6 & 46.322 & 15.194 & -0.0006566 \\
1.7 & 68.294 & 22.720 & -0.001570 \\
1.8 & 111.69 & 37.901 & -0.004014 \\
1.9 & 241.55 & 84.270 & -0.01020 \\
\hline \hline
\end{tabular}

gluon mass infrared (IR) regulator,

$$
\begin{array}{rlrl}
\Sigma_{1}^{\overline{\mathrm{MS}}} & =\frac{1}{2}, & V^{\overline{\mathrm{MS}}}=-\frac{25}{18}, \\
V_{D D}^{\overline{\mathrm{MS}}}=-\frac{121}{72}, & V_{\partial \partial}^{\overline{\mathrm{MS}}}=\frac{41}{72} .
\end{array}
$$

The lattice contributions are evaluated for domain-wall fermions with the Iwasaki gluon action $\left(c_{1}=-0.331\right)$, also choosing Feynman gauge and using a gluon mass IR regulator. $\Sigma_{1}$ has been evaluated in 65], while we calculated the vertex term $V$ for the first moment operator in [41]. Here we have calculated the vertex terms $V_{D D}$ and $V_{\partial \partial}$ for the second moment operator. Perturbative calculations with domain-wall fermions are explained in [64, 65] and the form of the Iwasaki gluon propagator can be found in [66]. Values for $\Sigma_{1}, V, V_{D D}$ and $V_{\partial \partial}$ are given as functions of $M$ in Table VI, along with $c, c_{D D}$

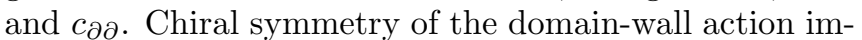
plies that these results also apply for the operators which are like those used here, but without the $\gamma_{5}$. We note that the perturbative renormalisation factor for the first moment operator using alternative fermion and gauge formulations can be found in 67] (domain-wall fermions and plaquette action), 68 (overlap fermions and LüscherWeisz action) and 69] (clover fermions and plaquette action). Second moment calculations with clover and Wilson fermions have been performed in 69] and 70] respectively (in both cases using the plaquette action).

Our numerical simulations use $M=1.8$. For this value of $M$, with the Iwasaki gluon action, the one-loop coefficient in the physical quark normalisation is $z_{w} \approx 112$ (extracted from $\Sigma_{w}$ in Table III of [65]), making it clear that mean-field improvement is necessary. We follow the prescription given in 65]. The first step is to define a mean-field value for the domain-wall height,

$$
M^{\mathrm{MF}}=M-4\left(1-P^{1 / 4}\right)=1.3029
$$

where $P=0.58813(4)$ is the average plaquette value in the chiral limit in our simulations. The physical quark normalisation factor becomes $\left[1-\left(w_{0}^{\mathrm{MF}}\right)^{2}\right] Z_{w}^{\mathrm{MF}}$, with

$$
\begin{aligned}
Z_{w}^{\mathrm{MF}} & =1+\frac{\alpha C_{\mathrm{F}}}{4 \pi} z_{w}^{\mathrm{MF}} \\
z_{w}^{\mathrm{MF}} & =\frac{2 w_{0}^{\mathrm{MF}}}{1-\left(w_{0}^{\mathrm{MF}}\right)^{2}}\left(\Sigma_{w}+32 \pi^{2} T_{\mathrm{MF}}\right)=5.2509,
\end{aligned}
$$

where $T_{\mathrm{MF}}=0.0525664$ [65] is a mean-field tadpole factor and $\Sigma_{w}$ is evaluated at $M^{\mathrm{MF}}$. Values for $z_{w}^{\mathrm{MF}}$ as a function of $M$ are quoted in Table VII] extracted from the results in [65]. Likewise, $\Sigma_{1}=3.9731, V=-4.1907$, $V_{D D}=-10.045$ and $V_{\partial \partial}=-0.1696$ are evaluated at $M^{\mathrm{MF}}$.

For the operator $\mathcal{O}_{D D}$ with two covariant derivatives, mean-field improvement introduces a factor $u_{\mathrm{pt}} / u$ where $u$ is the mean link (here taken to be $u=P^{1 / 4}$ ) and

$$
u_{\mathrm{pt}}=1-\frac{\alpha C_{\mathrm{F}}}{4 \pi} 8 \pi^{2} T_{\mathrm{MF}}
$$

is its perturbative expansion. For $\mathcal{O}_{\partial \partial}$ with two ordinary derivatives, in contrast, the extra factor is $u / u_{\mathrm{pt}}$. The mean-field-improved matching factors are thus

$$
\begin{aligned}
Z_{\mathcal{O}_{\{\rho \mu\}}}^{\mathrm{MF}} & =\frac{1}{1-\left(w_{0}^{\mathrm{MF}}\right)^{2}} \frac{1}{Z_{w}^{\mathrm{MF}}}\left[1+\frac{\alpha C_{\mathrm{F}}}{4 \pi}\left(-\frac{16}{3} \ln (\mu a)+c^{\mathrm{MF}}\right)\right] \\
Z_{D D, D D}^{\mathrm{MF}} & =\frac{1}{u} \frac{1}{1-\left(w_{0}^{\mathrm{MF}}\right)^{2}} \frac{1}{Z_{w}^{\mathrm{MF}}}\left[1+\frac{\alpha C_{\mathrm{F}}}{4 \pi}\left(-\frac{25}{3} \ln (\mu a)+c_{D D}^{\mathrm{MF}}-8 \pi^{2} T_{\mathrm{MF}}\right)\right] \\
Z_{D D, \partial \partial}^{\mathrm{MF}} & =u \frac{1}{1-\left(w_{0}^{\mathrm{MF}}\right)^{2}} \frac{1}{Z_{w}^{\mathrm{MF}}} \frac{\alpha C_{\mathrm{F}}}{4 \pi}\left(\frac{5}{3} \ln (\mu a)+c_{\partial \partial}^{\mathrm{MF}}\right)
\end{aligned}
$$

with $c^{\mathrm{MF}}=-0.6713, c_{D D}^{\mathrm{MF}}-8 \pi^{2} T_{\mathrm{MF}}=0.7408$ and $c_{\partial \partial}^{\mathrm{MF}}=0.7391$. To evaluate these expressions, we make two choices for the coupling. The first is a mean-field 
improved coupling defined using the measured plaquette value $P$, according to [1]

$$
\begin{aligned}
\frac{1}{g_{\mathrm{MF}}^{2}(\mu)}=\frac{P}{g_{0}^{2}}+d_{g}+ & c_{p}+\frac{22}{16 \pi^{2}} \ln (\mu a) \\
+ & N_{f}\left[d_{f}-\frac{4}{48 \pi^{2}} \ln (\mu a)\right]
\end{aligned}
$$

where $N_{f}$ is the number of dynamical quark flavours. For the Iwasaki gauge action with $c_{1}=-0.331$, the values $d_{g}=0.1053$ and $c_{p}=0.1401$ are given in [65], while values for $d_{f}$ as a function of $M$ were calculated in [71] and are quoted in Table VII In our simulations, $\beta=$ $6 / g_{0}^{2}=2.13$ with $N_{f}=3$ and $a^{-1}=1.729 \mathrm{GeV}$. The second choice is the continuum $\overline{\mathrm{MS}}$ coupling, calculated as outlined in Appendix A of [59]. At $\mu a=1$, we find $\alpha_{\mathrm{MF}}=0.1769$ and $\alpha^{\overline{\mathrm{MS}}}=0.3138$. We use these two values to evaluate the renormalisation factors above. We also evaluate the mean-field improved expression for the axial vector current renormalisation [65], interpolating to our mean-field $M^{\mathrm{MF}}$. The values are shown in Table VIII. The ratios of the renormalisation factors, from which the factor $1 /\left(1-\left(w_{0}^{\mathrm{MF}}\right)^{2}\right) Z_{w}^{\mathrm{MF}}$ cancels, are also shown in the table.

We take the mean value of the results with the two different choices for the coupling as the best answer for the renormalisation factors. The difference between the two choices will form the error. The relevant factors for the perturbative renormalisation of the ratios in Eqs. (25) and (28) are given in Table IX. Chiral symmetry here ensures that we do not have to distinguish between vector and axial-vector operators. We note that the contribution from the mixing term $Z_{D D, \partial \partial}$ is smaller than the error on $Z_{D D, D D}$ itself.

\section{B. Nonperturbative Renormalisation}

In order to renormalise the correlation functions nonperturbatively we make use of the Rome-Southampton $\mathrm{RI}^{\prime} / \mathrm{MOM}$ scheme 72] which we now briefly review and discuss refinements to [59]. The starting point and definition of the $\mathrm{RI}^{\prime} / \mathrm{MOM}$ scheme is a simple renormalisation condition that can be imposed independently of the regularisation used, thus on the lattice as well as in the continuum. This facilitates scheme changes which is important for the matching to $\overline{\mathrm{MS}}$. The renormalisation condition has the form

$$
\Lambda_{\mathcal{O}}(p)=\left.Z_{\mathcal{O}}(\mu) Z_{q}^{-1}(\mu) \Lambda_{\mathcal{O}}^{\text {bare }}(p)\right|_{p^{2}=\mu^{2}}=\Lambda_{\mathcal{O}}^{\text {tree }}(p),
$$

where $\Lambda_{\mathcal{O}}\left(\Lambda_{\mathcal{O}}^{\text {bare }}\right)$ is the renormalised (bare) vertex amplitude. Together with the quark field renormalisation $Z_{q}^{1 / 2}$, defined by $\psi=Z_{q}^{1 / 2} \psi^{\text {bare }}$, this defines the renormalisation constant $Z_{\mathcal{O}}$ for the operator $\mathcal{O}$. The renormalisation scale $\mu$ is set by the momentum of the external states entering the vertex amplitude. In the original
$\mathrm{RI}^{\prime} / \mathrm{MOM}$ scheme these momenta are exceptional, that is equal incoming and outgoing quark momenta, $p$ and $p^{\prime}$. For some renormalisation factors it is advantageous to use a non-exceptional symmetric choice of momenta $p^{2}=p^{\prime 2}=q^{2}$, where $q=p-p^{\prime}$, leading to the distinct RI/SMOM scheme. This suppresses unwanted infrared effects in the vertex amplitude, pion poles for example, and suggests a better-behaved accompanying continuum perturbation theory 73]. Exceptional momenta with $q=0$ also cause matrix elements of operators with total derivatives to vanish, making $Z_{D D, \partial \partial}$ inaccessible in our nonperturbative analysis.

The vertex amplitude is constructed from the unamputated Green's function

$$
\begin{aligned}
G_{\mathcal{O}}(p) & =\langle\psi(p) \mathcal{O}(0) \bar{\psi}(p)\rangle, \\
\mathcal{O}(0) & =\sum_{x, x^{\prime}} \bar{\psi}(x) J_{\mathcal{O}}\left(x, x^{\prime}\right) \psi\left(x^{\prime}\right) .
\end{aligned}
$$

The external quark lines need gauge fixing, for which we use Landau gauge. The current $J$ has the appropriate Dirac structure and may be non-local if the operator contains derivatives. For example, a single right derivative $\vec{D}_{\nu}$ in the vector case would correspond to

$$
J_{\mathcal{O}_{\rho \mu}}\left(x, x^{\prime}\right)=\gamma_{\rho} \frac{1}{2}\left(U\left(x, x^{\prime}\right) \delta_{x^{\prime}, x+\hat{\mu}}-U\left(x, x^{\prime}\right) \delta_{x^{\prime}, x-\hat{\mu}}\right)
$$

matching the definition in Eq. (18).

The vertex amplitude itself is found after amputating the Green's function and tracing with a suitable projector $P_{\mathcal{O}}$

$$
\Lambda_{\mathcal{O}}(p)=\operatorname{Tr}\left[\Pi_{\mathcal{O}}(p) P_{\mathcal{O}}\right]
$$

with

$$
\Pi_{\mathcal{O}}(p)=\langle S(p)\rangle^{-1}\left\langle G_{\mathcal{O}}(p)\right\rangle\langle S(p)\rangle^{-1} .
$$

We have used the quark propagator $S(p)$ and the angle brackets indicate the gauge average. The projector $P_{\mathcal{O}}$ depends on the particular operator and includes an overall normalisation factor to account for the colour and Dirac trace. In a simple example $P_{\mathcal{O}}$ would isolate the tree-level contribution to the vertex amplitude; we will detail our choices below. We have now defined the renormalisation procedure and will turn to details of the implementation before discussing the results.

\section{Momentum sources}

One refinement to our previous work [59] is the use of momentum sources [74]. In contrast to the point sources used before, this effectively amounts to a volume average over the lattice resulting in much smaller statistical errors 63]. Starting from (52) the Green's function in momentum space is

$$
G_{\mathcal{O}}(p)=\sum_{x, x^{\prime}}\left\langle\gamma_{5} S^{\dagger}(p)_{x} \gamma_{5} J_{\mathcal{O}}\left(x, x^{\prime}\right) S(p)_{x^{\prime}}\right\rangle
$$


TABLE VIII. Perturbative renormalisation factors and their ratios for two choices of the strong coupling, evaluated at $\mu a=1$.

\begin{tabular}{|c|c|c|c|c|c|c|c|}
\hline & $Z_{\mathcal{O}_{\{\rho \mu\}}}^{\mathrm{MF}}$ & $Z_{D D, D D}^{\mathrm{MF}}$ & $Z_{D D, \partial \partial}^{\mathrm{MF}}$ & $Z_{\mathrm{A}}^{\mathrm{MF}}$ & $\frac{Z_{\mathcal{O}_{\{\rho \mu\}}}^{\mathrm{MF}}}{Z_{\mathrm{A}}^{\mathrm{MF}}}$ & $\frac{Z_{D D, D D}^{\mathrm{MF}}}{Z_{\mathrm{A}}^{\mathrm{MF}}}$ & $\frac{Z_{D D, \partial \partial}^{\mathrm{MF}}}{Z_{\mathrm{A}}^{\mathrm{MF}}}$ \\
\hline$\overline{\alpha_{\mathrm{MF}}}$ & 0.9896 & 1.1604 & 0.0122 & 0.8009 & 1.2356 & 1.4488 & 0.0152 \\
\hline$\alpha^{\overline{\mathrm{MS}}}$ & 0.9162 & 1.0966 & 0.0202 & 0.6934 & 1.3214 & 1.5815 & 0.0291 \\
\hline
\end{tabular}

TABLE IX. Perturbative renormalisation factors to match the lattice results to $\overline{\mathrm{MS}}$ at $a \mu=1$.

\begin{tabular}{ccc}
\hline \hline$\frac{Z_{\mathcal{O}_{\{\rho \mu\}}}}{Z_{\mathrm{A}}}$ & $\frac{Z_{D D, D D}}{Z_{\mathrm{A}}}$ & $\frac{Z_{D D, \partial \partial}}{Z_{\mathrm{A}}}$ \\
\hline $1.28(4)$ & $1.52(7)$ & $0.022(7)$ \\
\hline \hline
\end{tabular}

where rather than use the quark propagator $S(x \mid y)$ obtained by inverting the Dirac Matrix $M$ on a point source

$$
\sum_{x} M\left(x^{\prime}, x\right) S(x \mid y)=\delta_{x^{\prime}, y} .
$$

we use $S(p)_{x}=\sum_{y} S(x \mid y) e^{\mathrm{i} p y}$ which can be found by inverting with a momentum source 74 ]

$$
\sum_{x} M\left(x^{\prime}, x\right) S(p)_{x}=e^{\mathrm{i} p x^{\prime}}
$$

and is defined on all lattice sites corresponding to the off-shell quarks used in the Green's function. The gain in statistical accuracy is paid for with a separate inversion for every momentum used in the simulation. However, this is more than compensated by a much reduced number of necessary configurations. Limiting ourselves to a few carefully chosen momenta, statistical fluctuations are reduced with lower overall computational cost.

The momenta we use are first of all constrained to be within a range $\Lambda_{\mathrm{QCD}} \ll p^{2} \ll 1 / a$ for the $\mathrm{RI}^{\prime} / \mathrm{MOM}$ scheme [72]. We use our previous results [59] to identify suitable values and focus on momenta which are expected to have reduced hypercubic lattice artefacts by trying to limit $\sum p_{\mu}^{4}$ for fixed $p^{2}$ [63] (see also [75, 76]). The values used are:

$$
\begin{aligned}
16^{3} \times 32: \quad & (1,1,2,3),(1,1,2,4),(1,2,2,1), \\
& (1,2,2,3),(1,2,2,4)
\end{aligned}
$$

and

$$
\begin{aligned}
24^{3} \times 64: \quad & (2,2,2,7),(2,2,2,8),(2,2,3,7), \\
& (2,2,3,8),(2,3,3,7),
\end{aligned}
$$

where we have given $n_{\mu}^{\mathrm{T}}$ for momenta $p_{\mu}=2 \pi n_{\mu} / L$ (with $L \rightarrow T$ for time components).

\section{2. $\quad$ Projectors}

We extend the set of operators considered previously in [59]. We now require operators with up to two deriva- tives, $\mathcal{O}_{\left\{\mu_{1} \ldots \mu_{n}\right\}}^{(5)}(n \leq 3)$, making the the necessary projectors $P_{\mathcal{O}}$ slightly more involved than for bilinears. Since we resort to readily available calculations [60, 61, 77] for the final conversion to $\overline{\mathrm{MS}}$ as well as to account for running, we have to tailor the projectors to match the $\mathrm{RI}^{\prime} / \mathrm{MOM}$ scheme and vertex functions used in the continuum calculations. Decomposing the amputated Green's function into terms allowed by Lorentz symmetry and remembering that we are taking all indices to be distinct, we find [60, 61, 77]

$$
G_{\mathcal{O}}(p)=\Sigma_{1}(p) \gamma_{\left\{\mu_{1}\right.} p_{\mu_{2}} \ldots p_{\left.\mu_{n}\right\}}+\Sigma_{2}(p) p_{\mu_{1}} \ldots p_{\mu_{n}} \not p .
$$

For simplicity we limit the discussion to the vector case here; axial-vector operators are analogous. The $\mathrm{RI}^{\prime} / \mathrm{MOM}$ scheme uses the contribution from $\Sigma_{1}(p)$ only in (59). The required projector $P_{\mathcal{O}}$ will depend on the momentum entering the Green's function and its (fixed) directions $\mu_{i}(i=1 \ldots n)$. In general, multiplying $G_{\mathcal{O}}$ with $\gamma_{\mu_{i}}$ picks up combinations of both terms $\Sigma_{1}$ and $\Sigma_{2}$. On the other hand, projecting with $\gamma_{\rho}$ where $\rho \notin\left\{\mu_{i}\right\}$ is only sensitive to $\Sigma_{2}$ (note that we have $n \leq 3$ ). Thus multiplying with the difference of the two Dirac matrices with appropriate normalisation and momentum factors ensures that the vertex amplitude in (54) contains $\Sigma_{1}(p)$ only. There are simpler special cases in which one or more components of the momentum $p$ are zero, causing the second term in (59) to vanish. However, since we tried to choose our momentum directions close to the diagonal of the lattice, we do not have momentum components that are zero.

For fixed indices $\mu_{i}(i=1 \ldots n)$ of the Green's function we can construct $n$ different projectors $P_{\mathcal{O}, i}$ by starting from any of the $\gamma_{\mu_{i}}$ :

$$
P_{\mathcal{O}, i}=\frac{\gamma_{\mu_{i}}-\gamma_{\rho} \frac{\bar{p}_{\mu_{i}}}{\bar{p}_{\rho}}}{\mathcal{N} \prod_{j \neq i, j=1}^{n} \bar{p}_{\mu_{j}}}, \text { with } i=1 \ldots n
$$

The normalisation $\mathcal{N}$ is chosen such that for the tree-level vertex amplitude we find $\Lambda_{\mathcal{O}}^{\text {tree }}(p)=1$. The index $\rho$ is different from any of the $\mu_{i}$ and such that its momentum component $\bar{p}_{\rho}$ is as small as possible to reduce discretisation errors. We use $\bar{p}_{\mu}=\sin p_{\mu}$ to better account for lattice momenta. The case of axial-vector operators $\mathcal{O}^{5}$ is straightforward, with $\gamma_{5}$ inserted in the appropriate places.

Combining the $n$ different $P_{\mathcal{O}, i}$ with the possible index combinations of the Green's functions results in a total of 
4, 12 and $12(n=1,2,3)$ choices to compute the vertex amplitude $\Lambda_{\mathcal{O}}(p)$ in Eq. (54), all of which should provide the same result for the final renormalisation constant in the absence of lattice artefacts. Because of the different sized momentum components in different lattice directions, the expected discretisation errors vary depending on the directions selected by the indices of the projector. We reflect these artefacts coming from breaking continuum $\mathrm{O}(4)$ symmetry to lattice hypercubic symmetry in the systematic error of our final results. With additional lattice spacings and the use of partially-twisted boundary conditions, we could eliminate hypercubic lattice artefacts in the continuum limit 78, 79].

\section{Quark field renormalisation}

In general, the renormalisation condition Eq. (51) requires knowledge of the field renormalisation $Z_{q}$ to obtain $Z_{\mathcal{O}}$. However, in the present calculation only ratios of renormalisation factors of operators with one, two or no derivatives appear, Eqs. (25) and (28). Combining this with our renormalisation condition leads to,

$$
\frac{Z_{\mathcal{O}, n=2,3}(\mu)}{Z_{\mathcal{O}, n=1}(\mu)}=\left.\frac{\Lambda_{\mathcal{O}, n=1}^{\text {bare }}(p)}{\Lambda_{\mathcal{O}, n=2,3}^{\text {bare }}(p)}\right|_{p^{2}=\mu^{2}}
$$

where the explicit $Z_{q}$ dependence drops out. As mentioned earlier, we can use either the vector or axial-vector bilinears in this ratio thanks to chiral symmetry. We follow our earlier procedure [59] and average $\Lambda_{\gamma_{\rho}}$ and $\Lambda_{\gamma_{\rho}}^{(5)}$ $\left(\Lambda_{V} / \Lambda_{A}\right.$ in the reference) to obtain our best answer. The analysis is also performed with $\Lambda_{\gamma_{\rho}}^{(5)}$ only and the difference of the two enters our systematic error.

\section{Results for renormalisation factors}

Compared to [59] the reduced statistical errors make previously hidden systematic effects apparent and quantifiable [63] and affect the way we extract the renormalisation factors. We start by considering different projectors for a fixed momentum $p_{\mu}$ of the external quarks, see Fig. 7 The results should be independent of the rotation and size of the momentum components used for the projector. The smaller statistical errors now reveal a disagreement due to lattice artefacts. We combine all choices for our best answer and account for the spread in our systematic error, improving previous estimates. Our general recipe to obtain the renormalisation factors follows. The ratio of bare vertex amplitudes is extrapolated linearly to the chiral limit $m_{q} \rightarrow-m_{\text {res }}$ for each momentum. Only in the chiral limit can we remove the running of our data points and match them to a continuum scheme. So by using [60, 61, 77] we take our results from the $\mathrm{RI}^{\prime} / \mathrm{MOM}$ scheme at scale $\mu^{2}=p^{2}$ to a common scale $\mu^{2}=4 \mathrm{GeV}^{2}$ and convert to $\overline{\mathrm{MS}}$ at that scale.

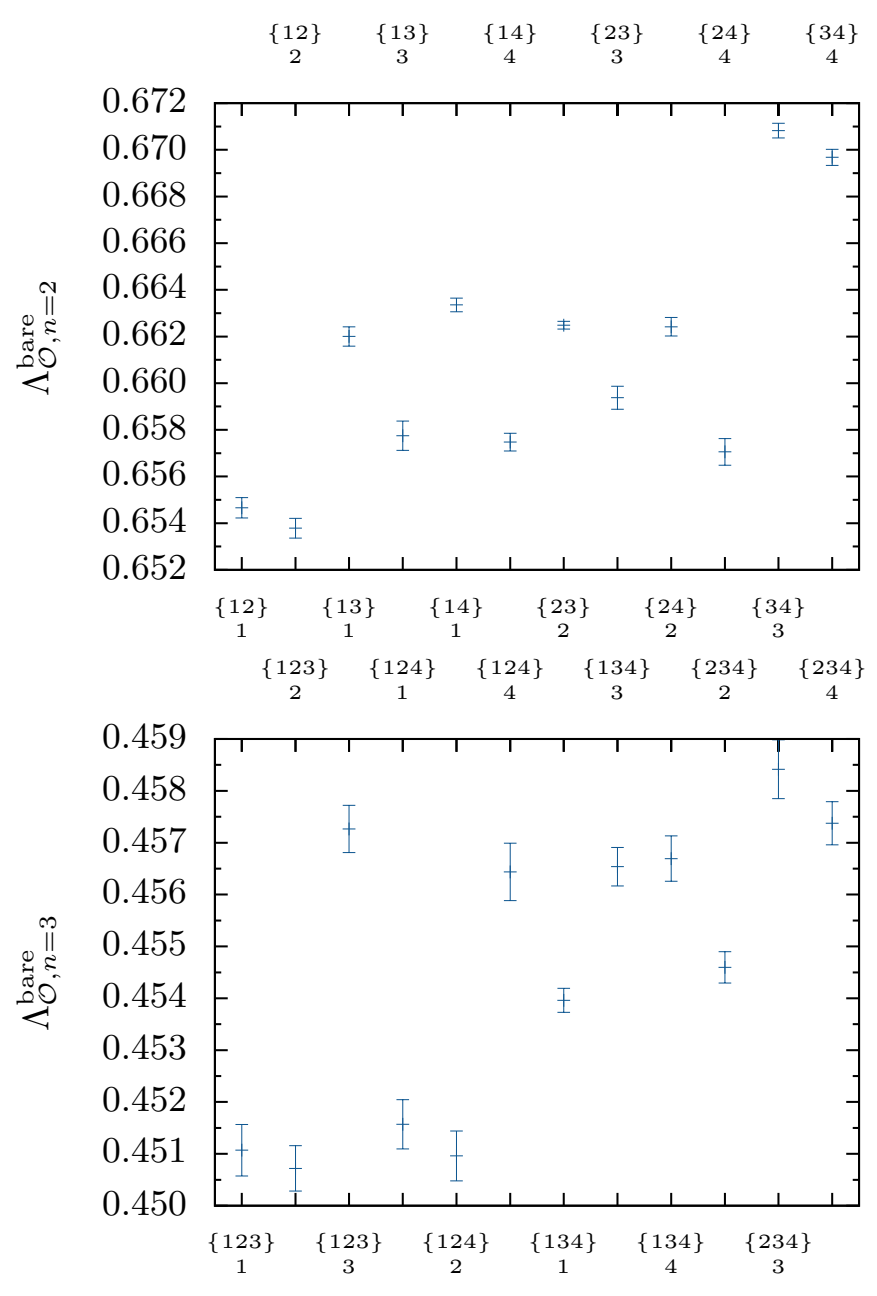

FIG. 7. Results for $\Lambda_{\mathcal{O}, n=2}^{\text {bare }}\left(\Lambda_{\mathcal{O}, n=3}^{\text {bare }}\right)$ on the top (bottom) for a fixed momentum $(a p)^{2}=1.78201, p^{T}=(2,2,3,8)$. The labels above and below the plots show the indices of the Green's function $\left\{\mu_{i}\right\}$ (top) and projector (bottom). The disagreement between the different projections is due to lattice artefacts.

The values thus obtained are then linearly interpolated to $p^{2}=(2 \mathrm{GeV})^{2}$ within our momentum window to obtain $Z_{\mathcal{O}, n=2,3} / Z_{\mathcal{O}, n=1}$ at a scale $\mu=2 \mathrm{GeV}$.

The central value is computed from the averaged values from all projectors and index combinations. A standard bootstrap analysis provides the statistical error which is

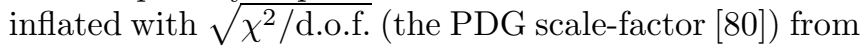
the interpolation. Several effects are taken into account for the systematic error. Lattice artefacts are the dominant effect. To estimate those, we perform the analysis for all projectors separately as indicated above and chose the highest and lowest result for each momentum for the interpolation. From the two fits, the larger deviation from the central value then constitutes the systematic error from discretisation effects (labelled 'spread' in the final table). This is a conservative approach for the discretisation error. Taking random choices of projectors (or rather their direction) for each momentum and look- 
ing at the $1 \sigma$ width of the range of results for many of those picks would lead to a smaller error. We account for missing higher order terms in the continuum perturbative calculation via the slope of the momentum interpolation, using the difference of our results at $p^{2}=(2 \mathrm{GeV})^{2}$ and $(0 \mathrm{GeV})^{2}$, indicated by 'slope', as a measure. We note, however, that we cannot disentangle perturbative and discretisation errors here and thus double count some of the discretisation effects. Another source of systematic error is the strange quark mass, kept fixed at $m_{s}=0.04$ in our simulation. We deal with that as described at the end of section IV.F in 59], estimating an error from half the linear dependence (slope) multiplied by the strange quark mass, $m_{\mathrm{s}}$. This error is labelled ' $\Delta m_{s}$ '. The last contribution to the systematic error is from the chiral symmetry breaking evident when comparing our vector and axial-vector operators [59, 73, 81]. This is estimated by the difference of the final results when taking the axialvector bilinear $(n=1)$ or the averaged vector and axialvector bilinear for the ratio in Eq. (61) (labelled ' $V-A$ '). Adding the four contributions in quadrature gives our systematic error.

To illustrate some of the steps mentioned above, we include in Fig. 8 two examples of the extrapolation to the chiral limit. Shown are extrapolations for all our five momenta, for the renormalisation factors for one and two derivatives. In Fig. 9] we show the renormalisation factors before and after we remove the running, again for one and two derivatives. Once at a common scale, the data points are much flatter indicating the validity of the scale conversion and the momentum window. Also included is the linear fit and our final result.

Our final values for the ratios of renormalisation factors are given in Table $\mathrm{X}$. These have been obtained using vector like operators. Results from the axial-vector operators are almost identical and show no low energy effects from breaking chiral symmetry, as for bilinears. We note that the renormalisation factors are significantly different from one and deviate substantially from the perturbative results. Thus nonperturbative renormalisation looks imperative here.

\section{Renormalised Results}

We now use the renormalisation factors from the previous section to convert our bare lattice results to $\overline{\mathrm{MS}}$ at $\mu=2 \mathrm{GeV}$. The local matrix elements in Eq. (9) require the renormalisation factors $Z_{\mathcal{O}_{\{\rho \mu\}}}, Z_{D D, D D}$ and $Z_{D D, \partial \partial}$ as defined in Eqs. (33) and (35). The first two are computed nonperturbatively while for the last one we use the perturbative result. From Eq. (35) we see that the mixing term requires the computation of a matrix element with an operator insertion of $\mathcal{O}_{\partial \partial}$. This is simplified since we use the ratios (25) and (28) to extract the moments of the distribution amplitudes. Within the ratios, the matrix element with the operator $\mathcal{O}_{\partial \partial}$ differs from the denominator only by the momentum factors and
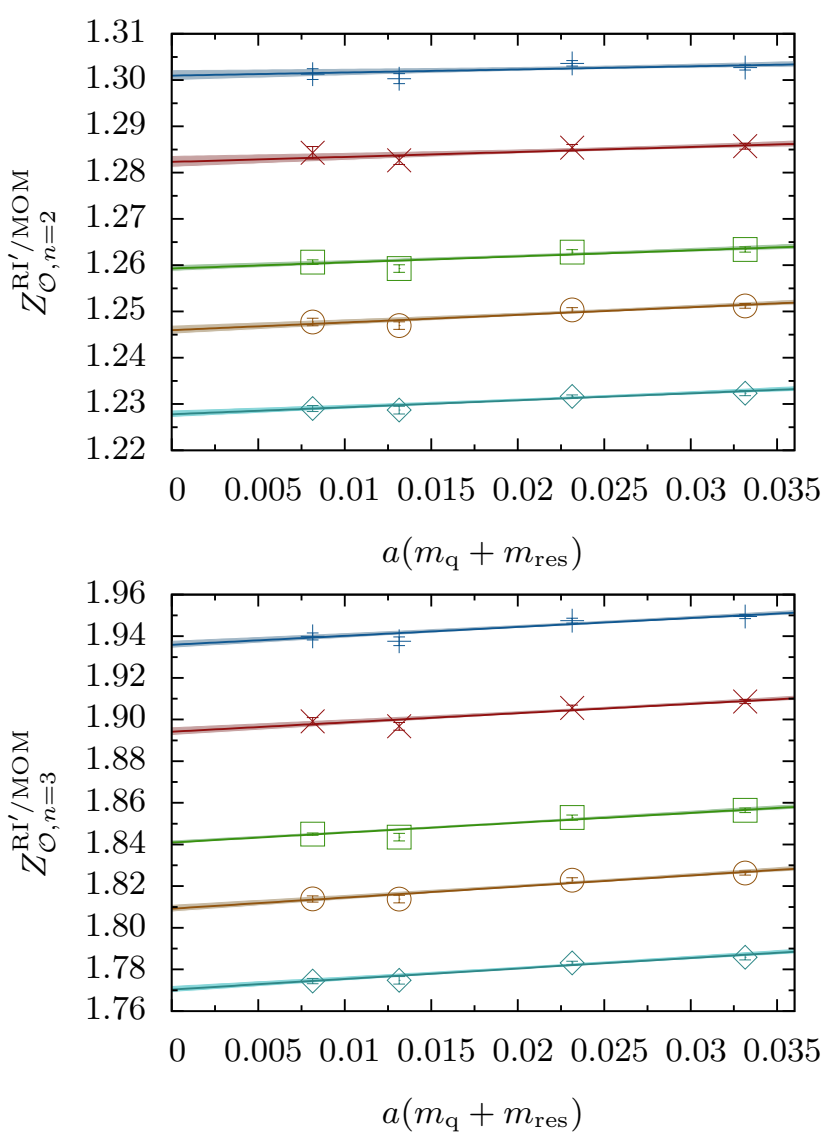

FIG. 8. Linear extrapolations of the renormalisation factors to the chiral limit. The top shows $Z_{\mathcal{O}, n=2}^{\mathrm{RI} / \mathrm{MOM}}$, the bottom plot is for $Z_{\mathcal{O}, n=3}^{\mathrm{RI}^{\prime} / \mathrm{MOM}}$. The momenta are increasing from top to bottom and we have $(a p)^{2}=1.2947,1.4392,1.6374,1.7820$ and 1.9801 .

thus does not have to be computed separately. It contributes a constant shift to the result. To summarise:

$$
\begin{aligned}
& \left\langle\xi^{1}\right\rangle^{\overline{\mathrm{MS}}}=\frac{Z_{\mathcal{O}_{\{\rho \mu\}}}}{Z_{A}}\left\langle\xi^{1}\right\rangle^{\text {bare }}, \\
& \left\langle\xi^{2}\right\rangle^{\overline{\mathrm{MS}}}=\frac{Z_{D D, D D}}{Z_{A}}\left\langle\xi^{2}\right\rangle^{\text {bare }}+\frac{Z_{D D, \partial \partial}}{Z_{A}} .
\end{aligned}
$$

With our best nonperturbative results from Table $\mathrm{X}$ and the perturbative result for the mixing term from Table IX (computed at the same scale of $\mu=2 \mathrm{GeV}$, $\left.\frac{Z_{D D, \partial \partial}}{Z_{A}}=0.027(8)\right)$, we arrive at the renormalised moments of the distribution amplitudes given in Table XI. The contribution from the mixing term in Eq. (62b) is small so using the perturbative result for $Z_{D D, \partial \partial}$ is not a drawback. Even if the correction of a nonperturbative result for $Z_{D D, \partial \partial}$ is as sizeable as for $Z_{\mathcal{O}_{\{\rho \mu\}}}$ or $Z_{D D, D D}$, the overall contribution remains comparable to our present error on $Z_{D D, D D} / Z_{A}$. Hence our results are essentially renormalised nonperturbatively. 
TABLE X. Final results for the renormalisation factors in $\overline{\mathrm{MS}}$ at $\mu=2 \mathrm{GeV}$. Results are given for both lattice sizes with all systematic errors. The perturbative results are also shown for comparison.

\begin{tabular}{l|cc|cc}
\hline \hline & \multicolumn{2}{|c|}{$Z_{\mathcal{O}_{\{\rho \nu\}} / Z_{A}}$} & \multicolumn{2}{c}{$Z_{D D, D D} / Z_{A}$} \\
& $16^{3} \times 32$ & $24^{3} \times 64$ & $16^{3} \times 32$ & $24^{3} \times 64$ \\
\hline central value & 1.54575 & 1.52893 & 2.06064 & 2.02800 \\
statistical error & 0.00249 & 0.00081 & 0.00482 & 0.00149 \\
spread & 0.02968 & 0.01809 & 0.03702 & 0.01534 \\
slope & 0.00470 & 0.00743 & 0.00097 & 0.02285 \\
$\Delta m_{s}$ & 0.00089 & 0.00232 & 0.00469 & 0.00992 \\
$V-A$ & 0.00723 & 0.00602 & 0.00938 & 0.00760 \\
total error & 0.03102 & 0.02061 & 0.03879 & 0.03026 \\
\hline \multicolumn{3}{l|}{ best result } \\
\multicolumn{2}{l|}{$1.5289(8)(206)$} \\
\hline
\end{tabular}

TABLE XI. Final results in the chiral limit in $\overline{\mathrm{MS}}$ at $\mu=2 \mathrm{GeV}$ for both of our lattice volumes. Here the first error is statistical, the second includes systematic errors from $m_{s}$, discretisation and renormalisation.

\begin{tabular}{c|ccccccc}
\hline \hline & $\left\langle\xi^{2}\right\rangle_{\pi}$ & $\left\langle\xi^{1}\right\rangle_{K}$ & $\left\langle\xi^{2}\right\rangle_{K}$ & $\left\langle\xi^{2}\right\rangle_{\rho}^{\|}$ & $\left\langle\xi^{1}\right\rangle_{K^{*}}^{\|}$ & $\left\langle\xi^{2}\right\rangle_{K^{*}}^{\|}$ & $\left\langle\xi^{2}\right\rangle_{\phi}^{\|}$ \\
\hline $16^{3} \times 32$ & $0.25(1)(2)$ & $0.035(2)(2)$ & $0.25(1)(2)$ & $0.25(2)(2)$ & $0.037(1)(2)$ & $0.25(1)(2)$ & $0.24(1)(1)$ \\
$24^{3} \times 64$ & $0.28(1)(2)$ & $0.036(1)(2)$ & $0.26(1)(2)$ & $0.27(1)(2)$ & $0.043(2)(3)$ & $0.25(2)(2)$ & $0.25(2)(1)$ \\
\hline \hline
\end{tabular}

\section{SUMMARY}

We have computed the first or first two lowest nonvanishing moments of the distribution amplitudes of the $\pi, K, K^{*}, \rho$ and $\phi$ mesons, using nonperturbative renormalisation of the lattice operators, with final numbers given in Table XI. Apart from the uncertainty in $m_{s}$ for the first moments, systematic errors mainly come from the renormalisation procedure. Within the current statistical errors on our data we do not see any finite size effects. With only one lattice spacing we can also only estimate a formal discretisation error of $O\left(a^{2} \Lambda_{\mathrm{QCD}}^{2}\right) \approx 4 \%$ from the $O(a)$-improved DWF action and operators; this is included in our sytematic error. The result for $\left\langle\xi^{1}\right\rangle_{K}$ in Table XI supercedes but is compatible with our earlier result in [41, 42], which was obtained on the $16^{3} \times 32$ ensembles only and used perturbative renormalisation.

Converting the lowest moment of the kaon distribution amplitude to the first Gegenbauer moment $a_{K}^{1}=$ $0.061(2)(4)$, we find it in agreement with sum rule results from Eq. (16) but with a much reduced uncertainty. We compare our results to those from the QCDSF Collaboration 23] in Table XII (preliminary results for the first moment of the vector meson distribution amplitudes are also available from QCDSF [27]). The results for $\left\langle\xi^{1}\right\rangle_{K}$ differ significantly. However, we observe that our measurements correspond to pion masses in the range 330$670 \mathrm{MeV}$ and are for $2+1$ dynamical flavours, whereas the QCDSF results are for pion masses around $600 \mathrm{MeV}$ and higher, with 2 dynamical flavours. For one data point from each collaboration where the pion and kaon masses are comparable, the $\left\langle\xi^{1}\right\rangle_{K}$ values differ by about one standard deviation. These points occur for the smallest values of $m_{K}^{2}-m_{\pi}^{2}$ from each collaboration; for larger values the points, and therefore slopes in $m_{K}^{2}-m_{\pi}^{2}$, differ. We plan to improve our results in the near future by reducing the systematic uncertainties. We will improve the nonperturbative calculation of the renormalisation factors by including the total derivative mixing term. We will also have an additional lattice spacing allowing us to estimate the continuum results, including using partiallytwisted boundary conditions to remove hypercubic lattice artefacts [78, 79]. Increased statistics on the $24^{3} \times 64$ lattice should also improve our conclusions about finite volume effects.

\section{ACKNOWLEDGMENTS}

The calculations reported here used the QCDOC computers [48, 82, 83] at Edinburgh University, Columbia University and Brookhaven National Laboratory (BNL). The Edinburgh QCDOC system was funded by PPARC JIF grant PPA/J/S/1998/00756 and operated through support from the Universities of Edinburgh, Southampton and Wales Swansea, and from STFC grant $\mathrm{PP} / \mathrm{E} 006965 / 1$. At BNL, the QCDOC computers of the RIKEN-BNL Research Center and the USQCD Collaboration were used. The software used includes: Chroma [54], QDP ++ and the CPS QCD codes [50], supported in part by the USDOE SciDAC program; the BAGEL [52] assembler kernel generator for many of the high-performance optimized kernels; and the UKHadron codes. We thank the University of Southampton for access to the Iridis computer system used in the calculations of the nonperturbative renormalisation factors (with support from STFC grant ST/H008888/1). DB, MAD, JMF, AJ, TDR and CTCS acknowledge support 
TABLE XII. Comparison to other lattice results (both for $\overline{\mathrm{MS}}$ at $\mu=2 \mathrm{GeV}$ ).

\begin{tabular}{l|cccc}
\hline \hline & $\left\langle\xi^{2}\right\rangle_{\pi}$ & $\left\langle\xi^{1}\right\rangle_{K}$ & $\left\langle\xi^{2}\right\rangle_{K}$ & $\left\langle\xi^{1}\right\rangle_{K^{*}}^{\|}$ \\
\hline this work $\left(24^{3} \times 64\right)$ & $0.28(1)(1)$ & $0.036(1)(2)$ & $0.26(1)(1)$ & $0.043(2)(3)$ \\
QCDSF [23] & $0.269(39)$ & $0.0272(5)$ & $0.260(6)$ & \\
\hline \hline
\end{tabular}
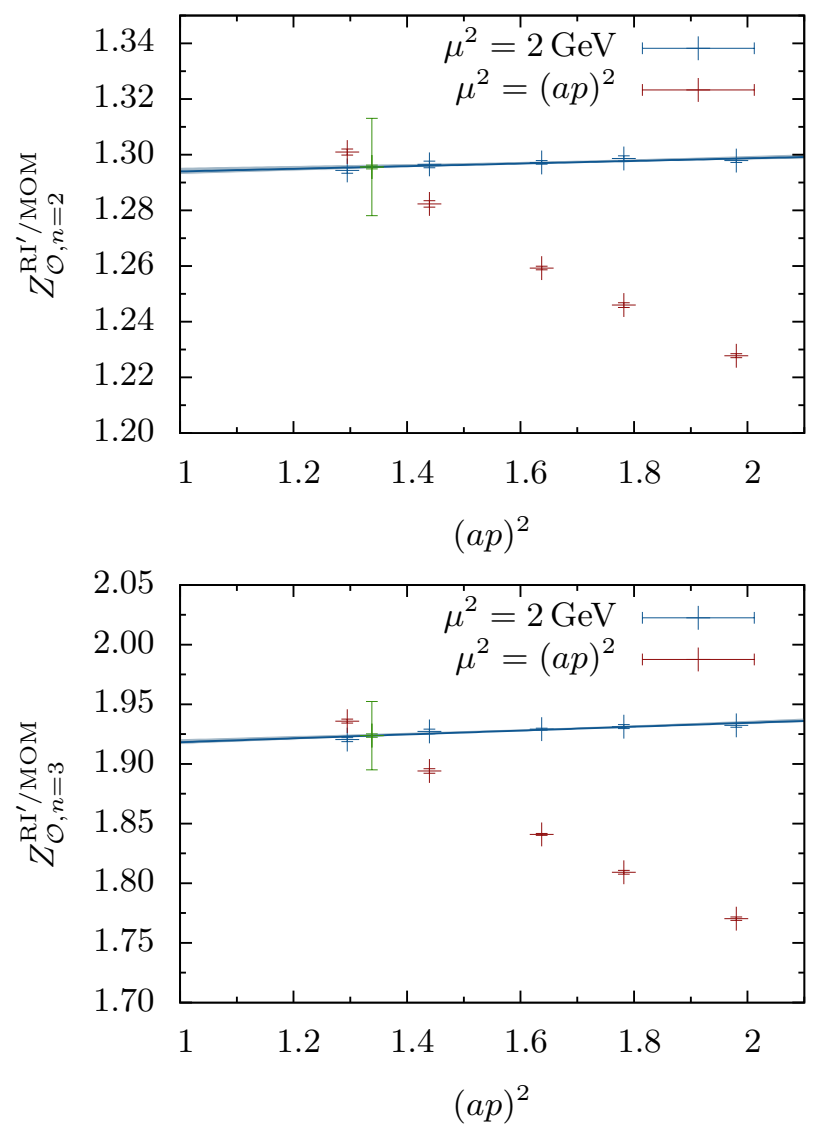

FIG. 9. These plots show the scale dependent $Z$ 's and $Z$ 's for a fixed scale $\mu=2 \mathrm{GeV}$ with the running successfully removed (both in $\mathrm{RI}^{\prime} / \mathrm{MOM}$ ). The top (bottom) plot is for the one (two) derivative case. Also included are the linear interpolation to the final result with the statistical error indicated by the error band. Our result at $\mu=2 \mathrm{GeV}$ is then shown with error bars for the statistical and systematic errors. from STFC Grant ST/G000557/1 and from EU contract MRTN-CT-2006-035482 (Flavianet); RA and PAB from STFC grants PP/D000238/1, PP/C503154/1 and ST/G000522/1; PAB from an RCUK Fellowship.
[1] G. P. Lepage and S. J. Brodsky, Phys. Rev. D22, 2157 (1980).

[2] P. Ball, V. M. Braun, Y. Koike, and K. Tanaka, Nucl. Phys. B529, 323 (1998), arXiv:hep-ph/9802299

[3] V. L. Chernyak, A. R. Zhitnitsky, and V. G. Serbo, JETP Lett. 26, 594 (1977).

[4] A. V. Efremov and A. V. Radyushkin, Phys. Lett. B94, 245 (1980)

[5] G. R. Farrar and D. R. Jackson, Phys. Rev. Lett. 43, 246 (1979)

[6] M. Beneke, G. Buchalla, M. Neubert, and C. T. Sachrajda, Phys. Rev. Lett. 83, 1914 (1999), hep-ph/9905312
[7] M. Beneke, G. Buchalla, M. Neubert, and C. T. Sachrajda, Nucl. Phys. B591, 313 (2000), arXiv:hep-ph/0006124

[8] M. Beneke, G. Buchalla, M. Neubert, and C. T. Sachrajda, Nucl. Phys. B606, 245 (2001), arXiv:hep-ph/0104110

[9] C. W. Bauer, S. Fleming, D. Pirjol, and I. W. Stewart, Phys. Rev. D63, 114020 (2001), arXiv:hep-ph/0011336.

[10] C. W. Bauer, D. Pirjol, and I. W. Stewart, Phys. Rev. D65, 054022 (2002), arXiv:hep-ph/0109045.

[11] C. W. Bauer, S. Fleming, D. Pirjol, I. Z. Rothstein, and I. W. Stewart, Phys. Rev. D66, 014017 (2002) 
arXiv:hep-ph/0202088

[12] V. M. Braun et al., Phys. Rev. D68, 054501 (2003) arXiv:hep-lat/0306006

[13] C. Allton et al. (RBC-UKQCD), Phys. Rev. D78, 114509 (2008). arXiv:0804.0473 [hep-lat].

[14] S. J. Brodsky, Y. Frishman, G. P. Lepage, and C. T. Sachrajda, Phys. Lett. B91, 239 (1980).

[15] V. M. Braun, G. P. Korchemsky, and D. Müller, Prog. Part. Nucl. Phys. 51, 311 (2003), arXiv:hep-ph/0306057.

[16] P. Ball and M. Boglione, Phys. Rev. D68, 094006 (2003) arXiv:hep-ph/0307337

[17] G. P. Lepage and S. J. Brodsky, Phys. Lett. B87, 359 (1979)

[18] A. V. Efremov and A. V. Radyushkin, Theor. Math. Phys. 42, 97 (1980).

[19] V. N. Gribov and L. N. Lipatov, Sov. J. Nucl. Phys. 15, 438 (1972).

[20] L. N. Lipatov, Sov. J. Nucl. Phys. 20, 94 (1975).

[21] Y. L. Dokshitzer, Sov. Phys. JETP 46, 641 (1977).

[22] G. Altarelli and G. Parisi, Nucl. Phys. B126, 298 (1977)

[23] V. M. Braun et al., Phys. Rev. D74, 074501 (2006), hep-lat/0606012

[24] A. Khodjamirian, T. Mannel, and M. Melcher, Phys. Rev. D68, 114007 (2003) arXiv:hep-ph/0308297 [hep-ph]

[25] J. Gronberg et al. (CLEO), Phys. Rev. D57, 33 (1998) arXiv:hep-ex/9707031.

[26] A. P. Bakulev, S. V. Mikhailov, and N. G. Stefanis, Fizika B13, 423 (2004), arXiv:hep-ph/0311140.

[27] V. M. Braun et al. (QCDSF-UKQCD), PoS LAT2007, 144 (2007), arXiv:0711.2174 [hep-lat]

[28] V. M. Braun et al. (QCDSF Collaboration), $\quad$ Phys. Rev. D79, 034504 (2009) arXiv:0811.2712 [hep-lat].

[29] G. Martinelli and C. T. Sachrajda, Phys. Lett. B190, 151 (1987)

[30] D. Daniel, R. Gupta, and D. G. Richards, Phys. Rev. D43, 3715 (1991).

[31] L. Del Debbio, M. Di Pierro, A. Dougall, and C. T. Sachrajda (UKQCD), Nucl. Phys. Proc. Suppl. 83, 235 (2000), arXiv:hep-lat/9909147.

[32] L. Del Debbio, M. Di Pierro, and A. Dougall, Nucl. Phys. Proc. Suppl. 119, 416 (2003), arXiv:hep-lat/0211037

[33] M. A. Shifman, A. I. Vainshtein, and V. I. Zakharov, Nucl. Phys. B147, 385 (1979).

[34] M. A. Shifman, A. I. Vainshtein, and V. I. Zakharov, Nucl. Phys. B147, 448 (1979)

[35] V. L. Chernyak and A. R. Zhitnitsky, Phys. Rept. 112, 173 (1984).

[36] P. Colangelo and A. Khodjamirian, in At the frontier of particle physics / Handbook of $Q C D$, edited by M. Shifman (World Scientific, Singapore, 2001) arXiv:hep-ph/0010175

[37] A. Khodjamirian, T. Mannel, and M. Melcher, Phys. Rev. D70, 094002 (2004), arXiv:hep-ph/0407226.

[38] V. M. Braun and A. Lenz, Phys. Rev. D70, 074020 (2004) arXiv:hep-ph/0407282,

[39] P. Ball and R. Zwicky, Phys. Lett. B633, 289 (2006), arXiv:hep-ph/0510338.

[40] P. Ball and R. Zwicky, JHEP 02, 034 (2006), arXiv:hep-ph/0601086.
[41] P. A. Boyle et al. (UKQCD), Phys. Lett. B641, 67 (2006), hep-lat/0607018

[42] P. Boyle, M. Donnellan, J. Flynn, A. Juttner, J. Noaki, et al., PoS LAT2006, 111 (2006), arXiv:hep-lat/0610025 [hep-lat]

[43] P. Hägler, $\quad$ Phys. Rept. 490, 49 (2010) arXiv:0912.5483 [hep-lat]

[44] M. Göckeler et al., Phys. Rev. D54, 5705 (1996) arXiv:hep-lat/9602029

[45] J. E. Mandula, G. Zweig, and J. Govaerts, Nucl. Phys. B228, 91 (1983).

[46] M. A. Clark and A. D. Kennedy, Phys. Rev. Lett. 98, 051601 (2007) arXiv:hep-lat/0608015

[47] P. Boyle, D. Chen, N. Christ, M. Clark, S. D. Cohen, et al., Nucl.Phys.Proc.Suppl. 129, 838 (2004) arXiv:hep-lat/0309096 [hep-lat]

[48] P. A. Boyle, C. Jung, and T. Wettig (QCDOC), ECONF C0303241, THIT003 (2003), hep-lat/0306023.

[49] P. Boyle, D. Chen, N. Christ, M. Clark, S. D. Cohen, et al., Nucl. Phys. Proc. Suppl. 140, 169 (2005).

[50] Columbia Physics System code developed by members of Columbia University, Brookhaven National Laboratory and UKQCD, http://qcdoc.phys.columbia.edu/cps.html.

[51] P. A. Boyle, Comp. Phys. Comm. 180, 2739 (2009).

[52] P. A. Boyle, (2005), Bagel assembler generator http://www.ph.ed.ac.uk/ paboyle/bagel/Bagel.html.

[53] C. Allton et al. (RBC and UKQCD), Phys. Rev. D76, 014504 (2007), arXiv:hep-lat/0701013.

[54] R. G. Edwards and B. Joo (SciDAC), Nucl. Phys. Proc. Suppl. 140, 832 (2005), arXiv:hep-lat/0409003

[55] P. Boyle (UKQCD), J. Comput. Phys. 179, 349 (2002) arXiv:hep-lat/9903033

[56] C. R. Allton et al. (UKQCD), Phys. Rev. D47, 5128 (1993), arXiv:hep-lat/9303009.

[57] J.-W. Chen and I. W. Stewart, $\quad$ Phys. Rev. Lett. 92, 202001 (2004), arXiv:hep-ph/0311285

[58] J.-W. Chen, H.-M. Tsai, and K.-C. Weng, Phys. Rev. D73, 054010 (2006), arXiv:hep-ph/0511036.

[59] Y. Aoki, P. Boyle, N. Christ, C. Dawson, M. Donnellan, et al., Phys. Rev. D78, 054510 (2008) arXiv:0712.1061 [hep-lat].

[60] J. A. Gracey, Nucl. Phys. B667, 242 (2003) arXiv:hep-ph/0306163

[61] J. A. Gracey, JHEP 10, 040 (2006), arXiv:hep-ph/0609231

[62] M. A. Donnellan et al., PoS LAT2007, 369 (2007), arXiv:0710.0869 [hep-lat]

[63] P. A. Boyle, D. Brömmel, M. A. Donnellan, J. M. Flynn, A. Jüttner, and C. T. Sachrajda (RBC and UKQCD Collaboration), PoS LATTICE2008, 165 (2008), arXiv:0810.1669 [hep-lat]

[64] S. Aoki, T. Izubuchi, Y. Kuramashi, and Y. Taniguchi, Phys. Rev. D59, 094505 (1999), arXiv:hep-lat/9810020.

[65] S. Aoki, T. Izubuchi, Y. Kuramashi, and Y. Taniguchi, Phys. Rev. D67, 094502 (2003), arXiv:hep-lat/0206013.

[66] Y. Iwasaki, "Renormalization group analysis of lattice theories and improved lattice action. 2. four-dimensional nonabelian SU $(N)$ gauge model," (1983), UTHEP-118.

[67] S. Capitani, Phys. Rev. D73, 014505 (2006) arXiv:hep-lat/0510091

[68] R. Horsley, H. Perlt, P. E. L. Rakow, G. Schierholz, 
and A. Schiller (QCDSF), Phys. Lett. B628, 66 (2005) arXiv:hep-lat/0505015.

[69] M. Göckeler et al., Eur. Phys. J. C48, 523 (2006) arXiv:hep-lat/0605002.

[70] M. Göckeler et al., Nucl. Phys. B717, 304 (2005) arXiv:hep-lat/0410009.

[71] S. Aoki and Y. Kuramashi, Phys. Rev. D68, 034507 (2003), arXiv:hep-lat/0306008

[72] G. Martinelli, C. Pittori, C. T. Sachrajda, M. Testa, and A. Vladikas, Nucl. Phys. B445, 81 (1995), hep-lat/9411010

[73] C. Sturm et al., Phys. Rev. D80, 014501 (2009) arXiv:0901.2599 [hep-ph]

[74] M. Göckeler et al., Nucl. Phys. B544, 699 (1999), arXiv:hep-lat/9807044.

[75] P. Boucaud, F. de Soto, J. Leroy, A. Le Yaouanc,
J. Micheli, et al., Phys. Lett. B575, 256 (2003), arXiv:hep-lat/0307026 [hep-lat]

[76] F. de Soto and C. Roiesnel, JHEP 0709, 007 (2007), arXiv:0705.3523 [hep-lat].

[77] J. A. Gracey, Nucl. Phys. B662, 247 (2003), arXiv:hep-ph/0304113

[78] R. Arthur and P. Boyle (RBC Collaboration, UKQCD Collaboration), (2010), arXiv:1006.0422 [hep-lat].

[79] R. Arthur and P. A. Boyle, (2010), arXiv:1010.6140 [hep-lat].

[80] K. Nakamura et al., J. Phys. G 37, 075021 (2010).

[81] Y. Aoki (RBC), PoS LATTICE2008, 222 (2008), arXiv:0901.2595 [hep-lat].

[82] P. Boyle et al., IBM Journal of Research and Development 49 (2/3), 351 (2005).

[83] P. A. Boyle et al., J. Phys. Conf. Ser. 16, 129 (2005). 